\title{
Untargeted Metabolomics Approach for the Discovery of Environment-Related Pyran-2-Ones Chemodiversity in a Marine-Sourced Penicillium restrictum
}

\author{
Van-Tuyen Le ${ }^{1}$, Samuel Bertrand ${ }^{1}$ (D), Thibaut Robiou du Pont ${ }^{1}$, Fabrice Fleury ${ }^{2} \mathbb{D}$, Nathalie Caroff $^{3}$, \\ Sandra Bourgeade-Delmas ${ }^{4}$, Emmanuel Gentil ${ }^{1}(\mathbb{D})$, Cedric Logé ${ }^{5}$, Gregory Genta-Jouve ${ }^{6}(\mathbb{D})$ and Olivier Grovel ${ }^{1, * \mathbb{D}}$
}

1 Faculty of Pharmacy, University of Nantes, EA 2160-Mer Molécules Santé, 9 rue Bias BP 53508, CEDEX 1, 44035 Nantes, France; van-tuyen.le1@univ-nantes.fr (V.-T.L.); samuel.bertrand@univ-nantes.fr (S.B.); thibaut.robiou@univ-nantes.fr (T.R.d.P.); emmanuel.gentil@univ-nantes.fr (E.G.)

2 Group of Mechanism and Regulation of DNA Repair and IMPACT Platform, UFIP UMR CNRS 6286, University of Nantes, 44322 Nantes, France; Fabrice.Fleury@univ-nantes.fr

3 Laboratoire EA3826, University of Nantes, IRS2 Nantes Biotech, CEDEX 1, 44100 Nantes, France; nathalie.caroff@univ-nantes.fr

4 UMR 125 PharmaDev, University of Toulouse, IRD, 31400 Toulouse, France; sandra.bourgeade-delmas@ird.fr

5 EA1155-IICIMED, University of Nantes, IRS2 Nantes Biotech, CEDEX 1, 44100 Nantes, France; Cedric.Loge@univ-nantes.fr

check for updates

Citation: Le, V.-T.; Bertrand, S.; Robiou du Pont, T.; Fleury, F.; Caroff, N.; Bourgeade-Delmas, S.; Gentil, E.; Logé, C.; Genta-Jouve, G.; Grovel, O. Untargeted Metabolomics Approach for the Discovery of Environment-

Related Pyran-2-Ones

Chemodiversity in a Marine-Sourced Penicillium restrictum. Mar. Drugs 2021, 19, 378. https://doi.org/ $10.3390 /$ md19070378

Academic Editors: Orazio Taglialatela-Scafati and Bill J. Baker

Received: 1 June 2021

Accepted: 26 June 2021

Published: 29 June 2021

Publisher's Note: MDPI stays neutral with regard to jurisdictional claims in published maps and institutional affiliations.

Copyright: (c) 2021 by the authors. Licensee MDPI, Basel, Switzerland. This article is an open access article distributed under the terms and conditions of the Creative Commons Attribution (CC BY) license (https:/ / creativecommons.org/licenses/by/ $4.0 /)$.
6 Laboratory Ecology, Evolution, Interactions of Amazonian Systems (LEEISA), USR 3456, University of French Guiana, CNRS Guyane, 275 Route de Montabo, 97334 Cayenne, France; gregory.genta-jouve@cnrs.fr

* Correspondence: olivier.grovel@univ-nantes.fr

Abstract: Very little is known about chemical interactions between fungi and their mollusc host within marine environments. Here, we investigated the metabolome of a Penicillium restrictum MMS417 strain isolated from the blue mussel Mytilus edulis collected on the Loire estuary, France. Following the OSMAC approach with the use of 14 culture media, the effect of salinity and of a musselderived medium on the metabolic expression were analysed using HPLC-UV/DAD-HRMS/MS. An untargeted metabolomics study was performed using principal component analysis (PCA), orthogonal projection to latent structure discriminant analysis (O-PLSDA) and molecular networking $(\mathrm{MN})$. It highlighted some compounds belonging to sterols, macrolides and pyran-2-ones, which were specifically induced in marine conditions. In particular, a high chemical diversity of pyran-2-ones was found to be related to the presence of mussel extract in the culture medium. Mass spectrometry (MS)- and UV-guided purification resulted in the isolation of five new natural fungal pyran-2-one derivatives-5,6-dihydro-6S-hydroxymethyl-4-methoxy-2H-pyran-2-one (1), (6S, 1'R, 2'S)-LL-P880 $\beta$ (3), 5,6-dihydro-4-methoxy-6S-(1'S, 2'S-dihydroxy pent-3'(E)-enyl)-2H-pyran-2-one (4), 4-methoxy-6$\left(1^{\prime} R, 2^{\prime} S\right.$-dihydroxy pent-3'(E)-enyl)-2H-pyran-2-one (6) and 4-methoxy-2H-pyran-2-one (7)-together with the known $\left(6 S, 1^{\prime} S, 2^{\prime} S\right)$-LL-P880 $\beta$ (2), (1'R, 2'S)-LL-P880 $\gamma$ (5), 5,6-dihydro-4-methoxy-2H-pyran2-one (8), (6S, 1'S , 2'R)-LL-P880 $\beta$ (9), (6S, 1'S)-pestalotin (10), 1'R-dehydropestalotin (11) and 6-pentyl4-methoxy-2H-pyran-2-one (12) from the mussel-derived culture medium extract. The structures of 1-12 were determined by 1D- and 2D-MMR experiments as well as high-resolution tandem MS, ECD and DP4 calculations. Some of these compounds were evaluated for their cytotoxic, antibacterial, antileishmanial and in-silico PTP1B inhibitory activities. These results illustrate the utility in using host-derived media for the discovery of new natural products.

Keywords: Penicillium restrictum; mussel-derived fungi; OSMAC; metabolome; pyran-2-one

\section{Introduction}

The phylum Mollusca is one of the largest and most diverse group in the animal kingdom. Estimated at 30,000 species in the sea, it comprises various bivalves such as 
oysters, mussels and clams [1]. As filter feeders, these shellfish can actively retain and concentrates particles and micro-organisms from their surrounding environment such as bacteria [2], viruses [3,4], protozoa [5], and fungi [6-8]. Zvereva et al. reported that fungal strains belonging to Aspergillus, Penicillium, Acremonium, and Alternaria genera are the most predominantly found in the two bivalve molluscs Crenomytilus grayanus and Modiolus modiolus [7]. Santos et al. reported eight fungal genera, such as Aspergillus, Penicillium, and Fusarium from the gills, intestine, and muscle tissue of the Nodipectennodosus scallop from marine farms in Brazil [8]. Sallenave-Namont et al. reported the presence of genera Penicillium, Trichoderma, Clasdosporium, Aspergillus, Paecilomyces along with Mucorales from mussel samples from marine shellfish farming areas [6]. In this way, all marine bivalves are colonized by a high diversity of microorganisms and fungi are major contributors to the microbiome of these holobionts. Various lifestyles such as symbiosis, parasitism, and mutualism have been described for fungi, depending on the species and the host $[9,10]$. In these ecosystems, fungi can establish numerous interactions with their hosts, mediated by secondary metabolites that serve as communication or chemical war purposes [10]. However, while the relationship between plants and endophytic fungi or between animals and fungal parasites have been the object of numerous works, almost no studies have investigated the chemical ecology of the association between mollusc bivalves and associated fungi.

Penicillium is one of the predominant genera in marine environments [11] and shellfishderived Penicillium strains have been demonstrated to produce a high range of metabolites. Some of these are identical to compounds from terrestrial origin such as roquefortine C [12], patulin, cladosporin, festuclavin or griseofulvin $[13,14]$, but others have been first detected from marine strains [10]. It seems that under the very specific conditions observed in marine environments, such as pressure, salinity or tides, some chemical pathways or chemical conditions are expressed that are not observed in terrestrial media. An interaction with marine invertebrates is one further condition that can be supposed to induce a dedicated chemistry. In this way, in a previous work, it was demonstrated that Penicillium strains isolated from shellfish produced more bioactive compounds than strains sampled from their surrounding environment $[15,16]$.

To access metabolites involved in the chemical communication between the two species and whose expression is silenced under usual laboratory culture conditions, culturebased strategies such as OSMAC (One Strain Many Compounds) are mandatory $[17,18]$. As part of this strategy, in vitro cultivation of fungi in the presence of host-derived substrates can induce specific metabolites. For example, the cultivation of a Penicillium hordei strain on tulip agar led to the medium-dependant production and isolation of the novel metabolites corymbiferone [19] and corymbiferan lactones A-D [20]. Host-derived media have also been successfully applied to enhance the yield of fungal inocula [21,22], to stimulate the production of low abundance metabolites [23], and to promote novel extracellular enzyme production and enhance protein expression [24-27]. In the marine field, the in vitro cultivation of fungi on mussel-derived substrates has been employed to convert agricultural and marine residues into microbial metabolites [27-32], and a recent study showed that mussel-processing wastewaters were a promising nutritive medium for astaxanthin production by the basidiomycetous species Xanthophyllomyces dendrorhous [29]. However, very little information is available on fungal metabolome expression induced by bivalves. Only one study has shown that the use of a mussel flesh-derived culture medium enhanced the production of cytotoxic metabolites by some mussel-derived fungi [16].

During our ongoing search for new marine fungal natural products, a Penicillium restrictum J.C. Gilman \& E.V. Abbott strain was isolated from a mussel sample in the Loire estuary, France. Although it is mainly considered as a typical terrestrial soil species, it has also been sampled from seawater, corals and marine sponges [33]. Penicillium restrictum isolates have been shown to produce some bioactive metabolites such as dehydrocarolic acid, gliotoxin [34], restricticin and its dimethyl derivative [35,36], curvularins [37], calbistrins [38,39], and the mycotoxins patulin and penicillic acid [40]. 
In this study, we present a metabolome investigation of a mussel-derived strain of $P$. restrictum MMS417, with a focus on environment-derived culture conditions. Alterations of culture conditions were performed following the OSMAC approach using seven culture media including a host-derived medium to evaluate the influence of mussel components on the production of specialized metabolites. In addition, the effect of salinity was also explored. Culture extracts were submitted to an untargeted metabolomics study using UPLC-IT/ToF-MS/MS-based molecular networking (MN) [41]. This resulted in highlighting some classes of metabolites overexpressed by the presence of mussel and seawater and to the MS-guided isolation of 12 pyran-2-ones including five new fungal natural products. Some of these compounds were tested for cytotoxic, antibacterial, antileishmanial activity and virtual PTP1B inhibition.

\section{Results}

\subsection{Effects of Culture Medium Components on the Metabolome of P. restrictum}

To explore the chemical diversity produced by P. restrictum MMS417 according to culture media, a complete metabolomics study was performed following the OSMAC approach. The strain was grown over six usual agar-based media-Czapek Yeast Agar (CYA), Dextrose Casein Agar (DCA), Potato Dextrose Agar (PDA), Yeast Extract Sucrose agar (YES), Malt Extract Agar (MEA) and Kohlmeyer-Medium Solid (KMS). A seventh medium was employed: Mussel Extract Sucrose Agar (MES), a host-derived medium [16] used to evaluate the influence of mussel flesh on the production of specific metabolites. Two different osmotic conditions were also used separately for each medium, by employing either distilled water (DW) or synthetic seawater (SSW) for the reconstitution of the media. Colony morphology was observed throughout the culture growth (Figure S1), showing that the morphology of the strain was dramatically modified according to each medium. A particularly unusual aspect was observed for cultures in the MES-SSW medium, which appeared to be "wet" and "submerged" at the centre of the colony with an absence of classical aerial mycelial structures.

Extracts of the cultures after 10 days were analysed by HPLC-HRMS using both the positive and negative ionisation modes. The chemical profiles of the 14 types of cultures (four replicates each) were converted to a data matrix using automated peak detection and compared by multivariate data analyses. The data were preliminarily analysed by principal component analysis (PCA) including quality control samples (QC), which confirmed data consistency (Pareto scaling) (Figure S2). In the positive ionization mode, the PCA scores plot achieved on all the 56 samples (Figure 1) showed a variation in the chemical profiles, with the first two components of PCA explaining $26.4 \%$ of the variance $(15 \%$ and $11.4 \%$ for PC1 and PC2, respectively).

It clearly appeared that both the medium composition and the presence of seawater influenced the extract composition. Extracts from media with the lowest content in nutrients (KMS, PDA and DCA) clustered closely together on the left part of the scores plot, while YES and MES extracts, issued from media for which the composition was similar except for the presence of mussel flesh in MES instead of yeast extract on YES, clustered in the other same part of the scores plot. On the other hand, DW and SSW extracts were separated from each other regardless of the composition of the medium. Of particular interest was the observation that MES-SSW extracts appeared clearly individualized from the other ones, suggesting that environment-based culture conditions can induce the production of specific compounds.

The influence of sea water/distilled water on the induction/repression of the production of metabolites was then globally investigated. For that purpose, a supervised orthogonal projection to latent structure discriminant analysis (OPLS-DA), comparing the two osmotic conditions was performed to highlight the distribution differences of metabolites in DW or SW extracts. 


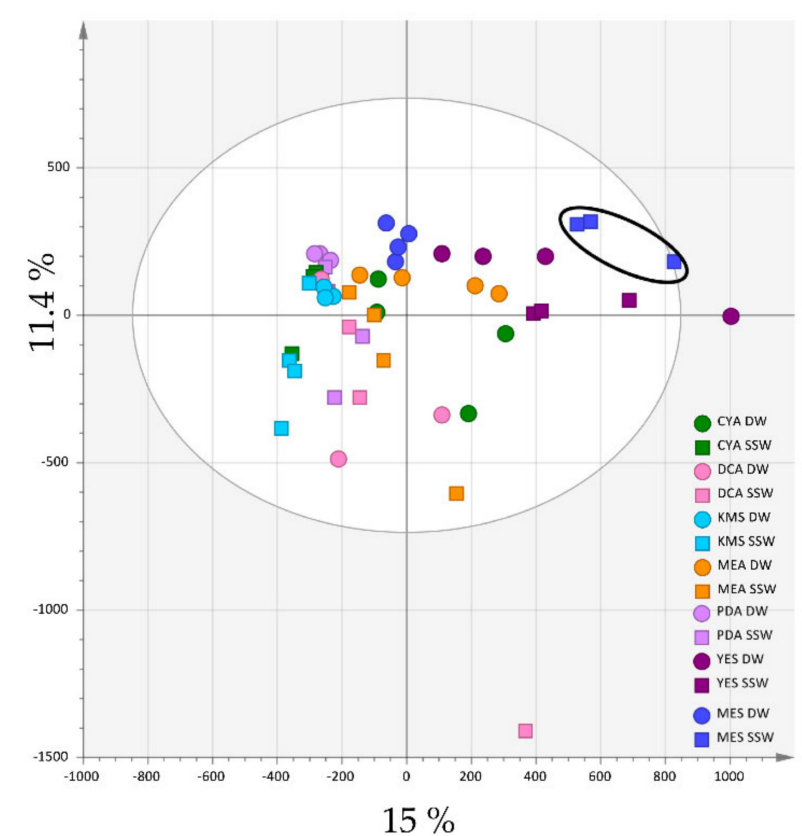

Figure 1. PCA (Pareto scaling) of the 14 Penicillium restrictum metabolic profiles (HPLC-HRMS data in positive ionization mode, $\mathrm{n}=4$ ). MES-SSW extracts are highlighted by a black circle.

The OPLS-DA (Figure 2a) showed significant discrimination between the two groups along the first dimension, and that the DW extracts metabolic profiles were less dispersed than for the SSW extracts on the orthogonal component. This was particularly obvious for MES-SSW and YES-SSW profiles which clustered separately from the other SSW extracts. They were also the most influenced by the type of water used. The S-loadings plot represented in Figure $2 \mathrm{~b}$ highlighted the metabolites distinct between the two groups. Features with a large Variable Influence on Projection values (VIP > 2) were considered to be the most contributing ones and were subjected to dereplication based on their (+)-HRESIMS spectrum, UV-vis spectrum and retention time leading to their putative annotation (Table 1). Nevertheless, many annotations could not be ascertained, as regioisomers and stereoisomers that can be produced simultaneously by the same fungal strain were described in the literature. Thus, these isobaric compounds were annotated as putative isomers of a reference compound already described from fungi. Most of the VIP compounds were found to correspond to some pyran-2-one derivatives, which could be clearly annotated due to the characteristic UV absorption of either the pyran-2-one $\left(\lambda_{\max }=280 \mathrm{~nm}\right)$ [42] or the 5,6-dihydropyran-2-one moieties $\left(\lambda_{\max }=238 \mathrm{~nm}\right)$ [43]. In SSW extracts, pestalotin and 2 '-hydroxypestalotin isomers [44,45] were highly produced, whereas dehydropestalotin, LL-P880 $\gamma$ [46], and 5,6-dihydro-4-methoxy-2H-pyran-2-one [45] were correlated with DW culture conditions. Various steroid derivatives were also detected as compounds distinctive of SSW extracts with antibiotic Mer-NF 8054A [47], 3 $\beta$-hydroxyergosta-8,14,24(28)-trien7-one [48] and paxisterol [49], together with the two macrolides melearoride A [50] and $\mathrm{N}$-demethylmelearoride A [51]. Among all the metabolites detected and annotated, only antibiotic TAN-1446A has been previously described from P. restrictum [52]. Some unidentified metabolites at $m / z 412.3335[\mathrm{M}+\mathrm{H}]^{+}(\mathrm{Rt}=23.28 \mathrm{~min})$ and $m / z 427.3221[\mathrm{M}+\mathrm{H}]^{+}$ $(\mathrm{Rt}=23.76 \mathrm{~min})$ were also highlighted in SSW profiles, which did not match to any compound in natural product databases (see Materials and Methods). 

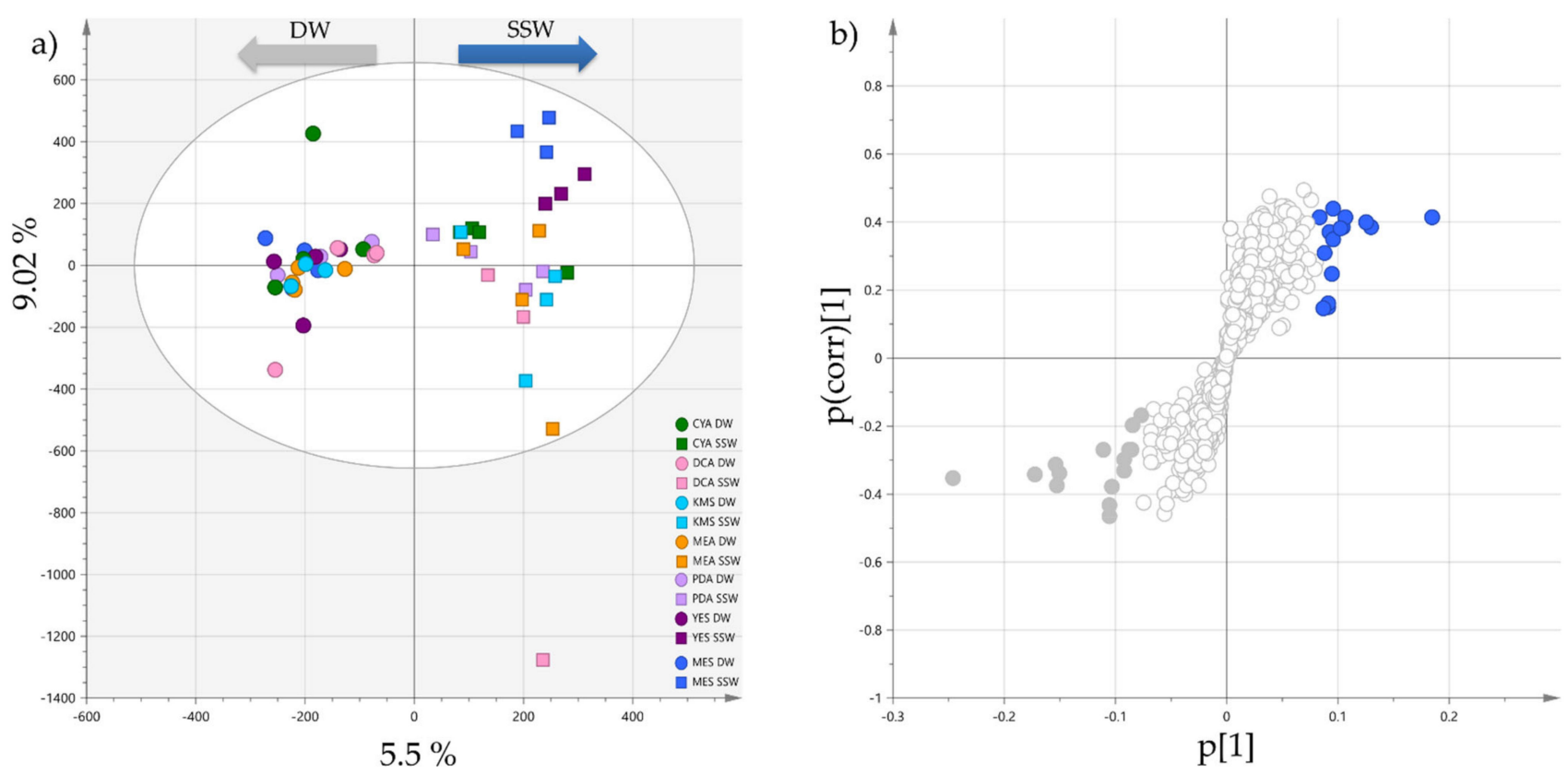

Figure 2. (a) OPLS-DA of the 14 Penicillium restrictum metabolic profiles (HPLC-HRMS data in positive ionization mode, $\mathrm{n}=4$ ) with separation of extracts following salinity (R2X cum 0.316 and R2Y cum 0.89, Q2 cum 0.766; CV-ANOVA $\left(p\right.$-value $\left.\left.=5.61 \times 10^{-11}\right)\right) ;(b)$ corresponding S-plot showing regions (blue: SSW; grey: DW) of highly specific features following salinity.

Table 1. Putative identification of characteristic ions highlighted in the OPLS-DA S-plot comparing media following salinity.

\begin{tabular}{|c|c|c|c|c|c|c|c|c|}
\hline & VIP & $m / z$ & $\underset{(\mathrm{min})}{\mathbf{R}_{\mathrm{t}}}$ & $\begin{array}{l}\text { Observed } \\
\text { Features } \\
\text { (Ionic } \\
\text { Species) }\end{array}$ & $\begin{array}{l}\text { Molecular } \\
\text { Formula } \\
\text { for M }\end{array}$ & $\Delta \mathrm{ppm}$ & $\begin{array}{c}\text { UV-vis } \\
\text { Absorption } \\
\left(\lambda_{\max } \mathrm{nm}\right)\end{array}$ & Putative Annotation \\
\hline \multirow{11}{*}{ 交 } & 4.05 & $\begin{array}{l}231.1225 \\
253.1050\end{array}$ & 6.52 & $\begin{array}{c}{[\mathrm{M}+\mathrm{H}]^{+}} \\
{[\mathrm{M}+\mathrm{Na}]^{+}}\end{array}$ & $\mathrm{C}_{11} \mathrm{H}_{18} \mathrm{O}_{5}$ & -3.24 & 238 & $2^{\prime}$-hydroxy pestalotin isomer \\
\hline & 3.46 & 411.3253 & 23.41 & {$[\mathrm{M}+\mathrm{H}]^{+}$} & $\mathrm{C}_{28} \mathrm{H}_{42} \mathrm{O}_{2}$ & -2.44 & n.d. & $3 \beta$-hydroxyergosta-8,14,24(28)-trien-7-one \\
\hline & 3.43 & 472.3422 & 22.27 & {$[\mathrm{M}+\mathrm{H}]^{+}$} & $\mathrm{C}_{29} \mathrm{H}_{45} \mathrm{NO}_{4}$ & -1.02 & n.d. & $N$-demethylmelearoride A \\
\hline & & 494.3197 & & {$[\mathrm{M}+\mathrm{Na}]^{+}$} & & & & \\
\hline & 3.15 & 486.3583 & 23.86 & {$[\mathrm{M}+\mathrm{H}]^{+}$} & $\mathrm{C}_{30} \mathrm{H}_{47} \mathrm{NO}_{4}$ & -0.07 & n.d. & Melearoride A \\
\hline & & 508.3407 & & {$[\mathrm{M}+\mathrm{Na}]^{+}$} & & & & \\
\hline & 2.8 & 443.3150 & 23.87 & {$[\mathrm{M}+\mathrm{H}]^{+}$} & $\mathrm{C}_{28} \mathrm{H}_{42} \mathrm{O}_{4}$ & -2.56 & n.d. & Paxisterol \\
\hline & 2.5 & 215.1281 & 9.08 & {$[\mathrm{M}+\mathrm{H}]^{+}$} & $\mathrm{C}_{11} \mathrm{H}_{18} \mathrm{O}_{4}$ & -1.09 & 237 & Pestalotin isomer \\
\hline & 2.42 & 412.3335 & 23.28 & {$[\mathrm{M}+\mathrm{H}]^{+}$} & $\mathrm{C}_{26} \mathrm{H}_{41} \mathrm{~N}_{3} \mathrm{O}$ & -1.53 & n.d. & - \\
\hline & 2.34 & 427.3221 & 23.76 & {$[\mathrm{M}+\mathrm{H}]^{+}$} & $\mathrm{C}_{28} \mathrm{H}_{42} \mathrm{O}_{3}$ & 2.06 & n.d. & - \\
\hline & 2.12 & 467.3157 & 19.62 & {$[\mathrm{M}+\mathrm{Na}]^{+}$} & $\mathrm{C}_{28} \mathrm{H}_{44} \mathrm{O}_{4}$ & 4.22 & n.d. & Antibiotic Mer-NF 8054A \\
\hline \multirow{15}{*}{ 方 } & 6.33 & 213.1139 & 9.82 & {$[\mathrm{M}+\mathrm{H}]^{+}$} & $\mathrm{C}_{11} \mathrm{H}_{16} \mathrm{O}_{4}$ & -2.78 & 279 & Dehydropestalotin isomer \\
\hline & & 235.0972 & & {$[\mathrm{M}+\mathrm{Na}]^{+}$} & & & & \\
\hline & 4.08 & 213.1132 & 10.03 & {$[\mathrm{M}+\mathrm{H}]^{+}$} & $\mathrm{C}_{11} \mathrm{H}_{16} \mathrm{O}_{4}$ & 2.42 & 237 & Pyran-2-one derivative \\
\hline & 3.89 & 307.1577 & 14.92 & {$[\mathrm{M}+\mathrm{H}]^{+}$} & $\mathrm{C}_{17} \mathrm{H}_{22} \mathrm{O}_{5}$ & 10.26 & n.d. & Antibiotic TAN 1446A \\
\hline & 3.88 & 213.1132 & 9.09 & {$[\mathrm{M}+\mathrm{H}]^{+}$} & $\mathrm{C}_{11} \mathrm{H}_{16} \mathrm{O}_{4}$ & -3.18 & 288 & Dehydropestalotin isomer \\
\hline & 3.82 & 251.0899 & 11.56 & {$[\mathrm{M}+\mathrm{Na}]^{+}$} & $\mathrm{C}_{11} \mathrm{H}_{16} \mathrm{O}_{5}$ & 1.42 & 275 & LL-P880 $\gamma$ isomer \\
\hline & & 267.0638 & & {$[\mathrm{M}+\mathrm{K}]^{+}$} & & & & \\
\hline & 3.72 & 129.0549 & 2.18 & {$[\mathrm{M}+\mathrm{H}]^{+}$} & $\mathrm{C}_{6} \mathrm{H}_{8} \mathrm{O}_{3}$ & -2.09 & 237 & 5,6-dihydro-4-methoxy-2H-pyran-2-one \\
\hline & 3.24 & 431.2787 & 22.98 & {$[\mathrm{M}+\mathrm{Na}]^{+}$} & $\mathrm{C}_{24} \mathrm{H}_{40} \mathrm{O}_{5}$ & 3.14 & n.d. & Aspergillus acid A \\
\hline & 2.97 & 515.2648 & 24.36 & {$[\mathrm{M}+\mathrm{H}]^{+}$} & $\mathrm{C}_{29} \mathrm{H}_{38} \mathrm{O}_{8}$ & 0.60 & n.d. & Citreohybridone B \\
\hline & 2.9 & 147.0654 & 2.13 & {$[\mathrm{M}+\mathrm{H}]^{+}$} & $\mathrm{C}_{6} \mathrm{H}_{10} \mathrm{O}_{4}$ & -2.27 & 237 & 5-hydroxy-3-methoxy-2-pentenoic acid \\
\hline & 2.79 & 379.3358 & 20.40 & {$[\mathrm{M}+\mathrm{H}]^{+}$} & $\mathrm{C}_{28} \mathrm{H}_{42}$ & -1.78 & n.d. & 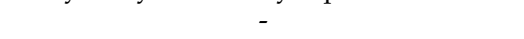 \\
\hline & 2.58 & 185.0845 & 5.88 & {$[\mathrm{M}+\mathrm{H}]^{+}$} & $\mathrm{C}_{12} \mathrm{H}_{11} \mathrm{NO}$ & 2.36 & n.d. & - \\
\hline & 2.22 & 544.3654 & 18.59 & {$[\mathrm{M}+\mathrm{H}]^{+}$} & $\mathrm{C}_{32} \mathrm{H}_{49} \mathrm{NO}_{6}$ & 2.91 & n.d. & Pestalotiopin B \\
\hline & 2.11 & 520.3413 & 16.45 & {$[\mathrm{M}+\mathrm{Na}]^{+}$} & $\mathrm{C}_{31} \mathrm{H}_{47} \mathrm{NO}_{4}$ & 1.96 & n.d. & - \\
\hline
\end{tabular}


To observe the specific effect of the mussel flesh on the induction of metabolites in P. restrictum, an OPLS-DA was carried out comparing the MES-SSW extracts to other SSW extracts (Figure 3). The validated OPLS-DA model (Figure 3, R2Y cum 0.89, Q2 cum 0.766 ) exhibited a significant discrimination between the two groups following the first component, the MES-SSW extracts being well separated from the others. The Splot showed that the ions responsible for distinction of MES-SSW (VIP > 3) were still pyran-2-one derivatives: isomers of pestalotin, 2'-hydroxypestalotin, dehydroxypestalotin and LL-P880 $\gamma$ were dereplicated among the compounds with the highest VIP values (Table 2). The ion at $m / z 211.0581[\mathrm{M}+\mathrm{Na}]^{+}$eluted at $6.58 \mathrm{~min}$ remained unidentified when searched in fungal natural products databases. However, its UV-Vis spectrum showed an absorption band at $\lambda_{\max }=237 \mathrm{~nm}$, which is characteristic of a 5,6-dihydropyran-2-one moiety [43]. The compound at $m / z 147.0654$ was annotated as 5-hydroxy-3-methoxy-2pentenoic acid (verrucolone or arabenoic acid), which corresponds to an open acidic form of 5,6-dihydro-4-methoxy-2H-pyran-2-one, two compounds which have been previously described from Penicillium strains $[53,54]$. From this OPLS-DA, it could also be noticed that on the first orthogonal component, which corresponds to an unsupervised direction, the YES-SSW extracts were clearly distinguished from the other SSW extracts. Another OPLSDA (Figure S3, Table S1) was conducted comparing these extracts to other SSW extracts, which showed that other pyran-2-ones together with melearorides were responsible for this discrimination.
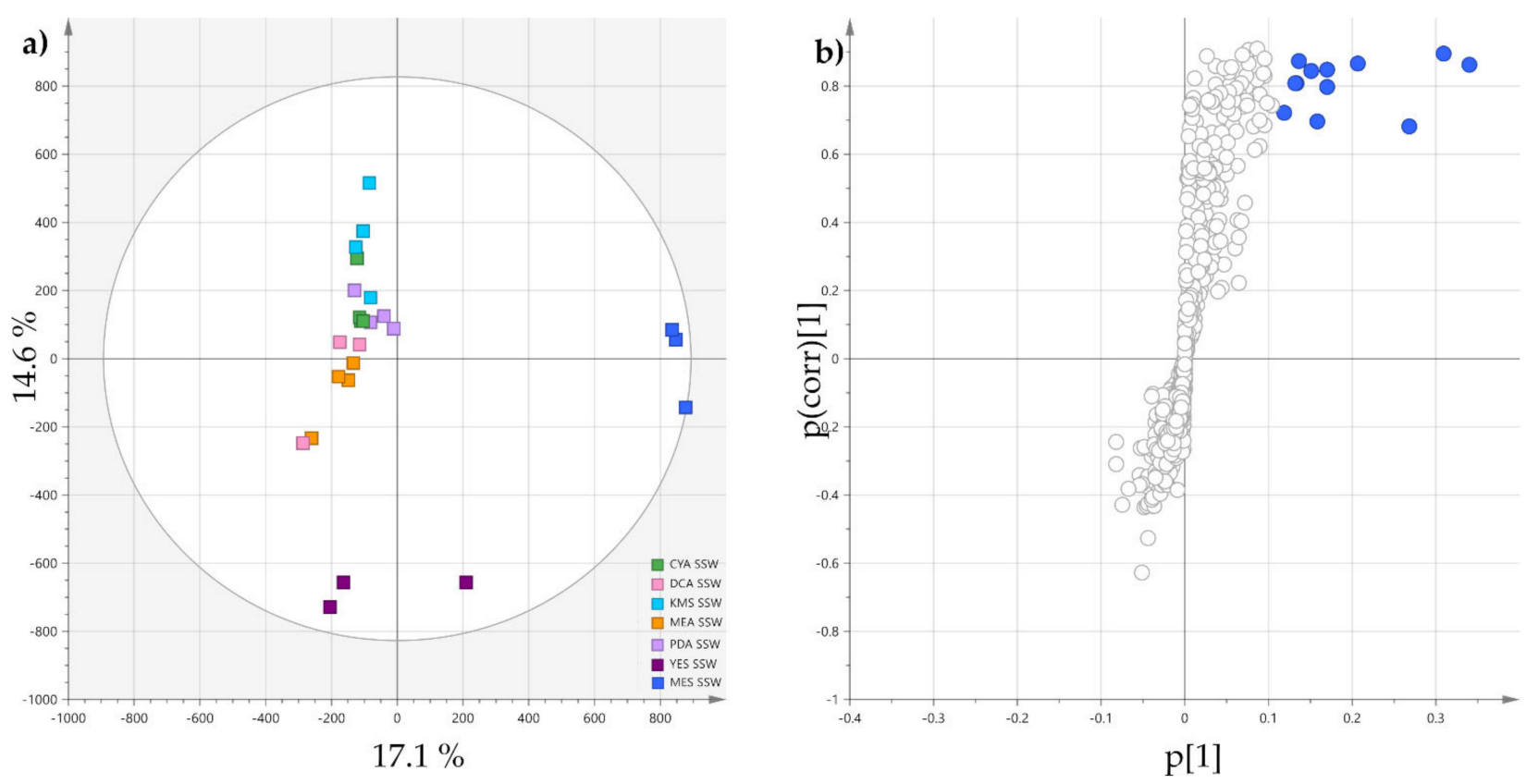

Figure 3. (a) OPLS-DA scores-plot of MES-SSW extracts versus the six other SSW extracts (positive ionization, $\mathrm{n}=4$; R2X cum 0.317 and R2Y cum 0.927, Q2 cum 0.777; CV-ANOVA ( $p$-value $\left.=2.72 \times 10^{-6}\right)$ ); (b) corresponding S-plot: features highlighted in blue correspond to discriminatory ions with VIP $>3$. 
Table 2. Identification of characteristic ions highlighted in the OPLS-DA S-plot comparing MES-SSW extracts to other SSW extracts.

\begin{tabular}{|c|c|c|c|c|c|c|c|}
\hline VIP & $m / z$ & $\mathbf{R}_{\mathrm{t}}(\min )$ & $\begin{array}{c}\text { Observed } \\
\text { Features } \\
\text { (Ionic Species) }\end{array}$ & $\begin{array}{l}\text { Molecular } \\
\text { Formula } \\
\text { for } \mathrm{M}\end{array}$ & $\Delta \mathrm{ppm}$ & $\begin{array}{c}\text { UV-vis } \\
\text { Absorption } \\
\left(\lambda_{\max } \mathrm{nm}\right)\end{array}$ & Putative Annotation \\
\hline 8.95 & $\begin{array}{l}231.1225 \\
253.1050 \\
269.0800 \\
483.2219\end{array}$ & 6.52 & $\begin{array}{c}{[\mathrm{M}+\mathrm{H}]^{+}} \\
{[\mathrm{M}+\mathrm{Na}]^{+}} \\
{[\mathrm{M}+\mathrm{K}]^{+}} \\
{[2 \mathrm{M}+\mathrm{Na}]^{+}}\end{array}$ & $\mathrm{C}_{11} \mathrm{H}_{18} \mathrm{O}_{5}$ & -3.24 & 238 & 2'-hydroxypestalotin isomer \\
\hline 7.95 & 211.0581 & 6.58 & {$[\mathrm{M}+\mathrm{Na}]^{+}$} & $\mathrm{C}_{8} \mathrm{H}_{12} \mathrm{O}_{5}$ & -0.68 & 237 & Unknown pyran-2-one derivative \\
\hline 5.45 & 213.1139 & 9.82 & {$[\mathrm{M}+\mathrm{H}]^{+}$} & $\mathrm{C}_{11} \mathrm{H}_{16} \mathrm{O}_{4}$ & 5.71 & 279 & Dehydroxypestalotin isomer \\
\hline 4.59 & 129.0549 & 2.18 & {$[\mathrm{M}+\mathrm{H}]^{+}$} & $\mathrm{C}_{6} \mathrm{H}_{10} \mathrm{O}_{4}$ & -2.09 & 237 & $\begin{array}{l}\text { 5,6-dihydro-4-methoxy- } 2 \mathrm{H} \text { - } \\
\text { pyran-2-one }\end{array}$ \\
\hline 4.53 & $\begin{array}{l}215.1281 \\
237.1117\end{array}$ & 9.08 & $\begin{array}{c}{[\mathrm{M}+\mathrm{H}]^{+}} \\
{[\mathrm{M}+\mathrm{Na}]^{+}}\end{array}$ & $\mathrm{C}_{11} \mathrm{H}_{18} \mathrm{O}_{4}$ & -1.09 & 237 & Pestalotin isomer \\
\hline 3.57 & 251.0905 & 6.68 & {$[\mathrm{M}+\mathrm{Na}]^{+}$} & $\mathrm{C}_{11} \mathrm{H}_{16} \mathrm{O}_{5}$ & 5.8 & 275 & $\begin{array}{c}\text { LL-P880 } \gamma \text { isomer } \\
\text { 5-hydroxy-3-methoxy-2- }\end{array}$ \\
\hline 3.50 & 147.0654 & 2.13 & {$[\mathrm{M}+\mathrm{H}]^{+}$} & $\mathrm{C}_{6} \mathrm{H}_{10} \mathrm{O}_{4}$ & -4.99 & 237 & $\begin{array}{c}\text { pentenoic } \\
\text { acid }\end{array}$ \\
\hline 3.24 & 251.0905 & 11.56 & {$[\mathrm{M}+\mathrm{Na}]^{+}$} & $\mathrm{C}_{11} \mathrm{H}_{16} \mathrm{O}_{5}$ & 1.82 & 275 & LL-P880 $\gamma$ isomer \\
\hline
\end{tabular}

\subsection{Molecular Networking of P. restrictum MES-SSW Extract}

A molecular network (MN, Figure 4) of the MES-SSW extract was constructed using LC-HRMS/MS data obtained in the positive ionization mode, including the retention time of all detected ions in order to distinguish the isobaric compounds. The MN consisted of 83 nodes representing the most abundant ions in the chemical profile, which were grouped into seven clusters. Compounds significantly discriminating the MESS-SSW extract on the OPLS-DA were searched among the nodes and mapped on the MN. Among a total of 43 nodes corresponding to compounds for which VIP $>1$ (light blue nodes), seven of the ions with VIP $>3$ (dark blue nodes) were present in the MN. Five compounds were not detected due to a too low relative abundance in the extract to be fragmented using the MS/MS parameters. The largest cluster (Figure 4a) corresponded to ergosterol derivatives, some of them being dereplicated by the GNPS database as ergostapentaene $(m / z 375.3014)$, ergostahexaene $(m / z$ 377.3163), ergostatetraen-3-one $(m / z$ 393.3115), ergostatetraen-3-ol $(m / z$ 395.3301) and hydroxyergostatrien-7-one $(\mathrm{m} / \mathrm{z}$ 411.3253) analogues [48], among which five were overproduced in the mussel-derived medium. A more detailed list of annotated metabolites can be found in Table S2. In the second cluster (Figure 4b), the node

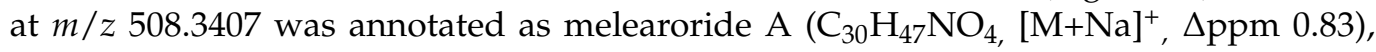
a 13-membered macrolide previously isolated from a marine-derived Penicillium meleagrinum var. viridiflavum [50]. Four other melearoride derivatives-PF 1163E $(m / z$ 536.3657, $\left.[\mathrm{M}+\mathrm{Na}]^{+}, \mathrm{C}_{32} \mathrm{H}_{51} \mathrm{NO}_{4} \Delta \mathrm{ppm}-10.96\right)$ [55], PF 1163B ( $/ z / z$ 484.3053, [M+Na] $]^{+}, \mathrm{C}_{27} \mathrm{H}_{43} \mathrm{NO}_{5}$ $\Delta$ ppm 2.91) [56,57], $N$-demethylmelearoride A $\left(m / z\right.$ 472.3422, [M+H] ${ }^{+}, \mathrm{C}_{29} \mathrm{H}_{45} \mathrm{NO}_{4} \Delta \mathrm{ppm}$ -1.02) [51] - were dereplicated in the same cluster, for which the annotation was confirmed by a thorough manual interpretation of their MS/MS fragmentation pattern (Figure S4). Two nodes at $m / z 522.3549$ and 522.3563 were connected with the $m / z 536.3657$ node with a difference of 14.01 suggesting that they corresponded to two undescribed demethyl derivatives. Two other nodes at $m / z 492.3083,746.5283$ corresponding to unknown compounds were also strongly connected within this cluster, showing a high diversity of new compounds in this rare macrolide family, which were specifically produced by the fungus on YES-SSW and MES-SSW media. 

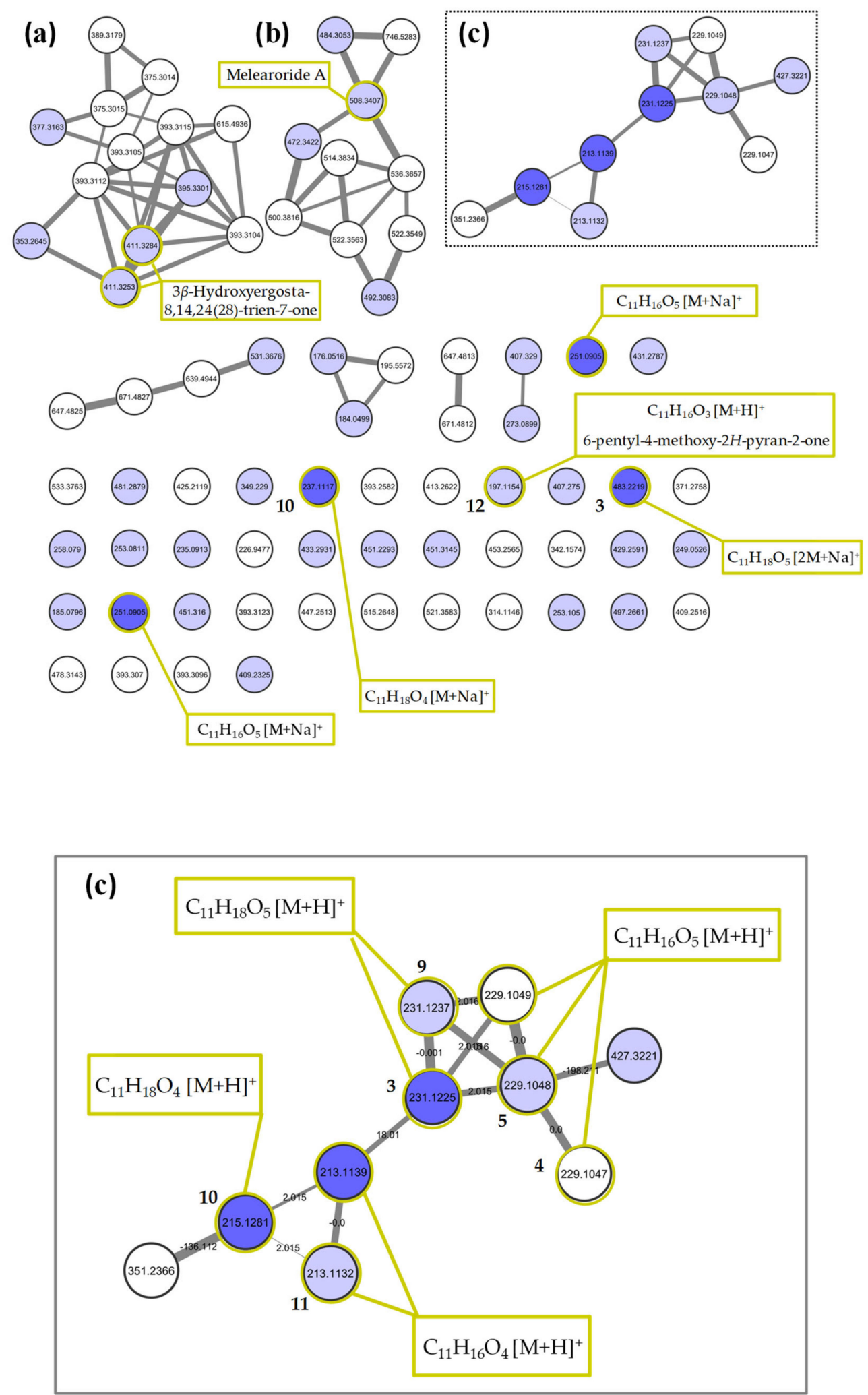

Figure 4. MS/MS molecular network obtained from the MES-SSW extract of Penicillium restrictum MMS417. Above: complete network showing the three main clusters, (a) ergosterol derivatives, (b) melearorides derivatives, (c) pyran-2-ones. Below: expansion of pyran-2-one cluster (c). Light blue nodes represent features with VIP $>1$, dark blue nodes represent features with VIP > 3. Bold numbers highlight isolated compounds. 
The third cluster (Figure 4c) was composed of 10 nodes related to pyran-2-one compounds, linked together by an accurate mass difference of 2.01 or 18.01. Three of them were some of the ions highlighted in the OPLS-DA with VIP > 3: $m / z 215.1281$ (pestalotin isomer), $m / z 213.1139$ (dehydropestalotin isomer) and $m / z 231.1225$ (2'-hydroxypestalotin isomer). Among the other nodes, four exhibited a VIP score between 1 and 3 . Two of them were dereplicated as other analogs of dehydroxypestalotin and 2'-hydroxypestalotin, and the node at $m / z 229.1048$ was consistent with LL-P880 $\gamma$. Two other nodes with the same accurate mass and similar fragmentation pattern (cosine score $=0.70$ and 0.86 ) were linked to the latter but with a different retention time, showing that three different isomers of LL-P880 $\gamma$ were present. Some of the ions with VIP $>3$ in the O-PLSDA model did not appear to be connected to this pyran-2-one cluster because they were observed as $[\mathrm{M}+\mathrm{Na}]^{+}$ adducts and thus exhibited different fragmentation patterns than the corresponding protonated molecule. In particular, two other LL-P880 $\gamma$ isomers were observed as isolated nodes at $m / z$ 251.0905. Other pyran-2-ones were manually searched among the self-loop nodes using their UV-vis absorbance spectra, on the basis of the characteristic absorbance band at $280 \mathrm{~nm}$ revealing a pyran-2-one ring and $238 \mathrm{~nm}$ for the 5,6-dihydropyran-2-one moiety. A node at $m / z$ 197.1154 [M+H] $]^{+}$was then annotated as 6-pentyl-4-methoxy-2H-pyran-2-one $\left(\lambda_{\max } 280 \mathrm{~nm}\right)$, a dehydroxy derivative of dehydropestalotin [12]. Manual curation of its MS/MS spectrum and comparison with those of other pyran-2-ones confirmed this annotation as it did not present an $[\mathrm{M}-18+\mathrm{H}]^{+}$fragment ion characteristic of the hydroxyl group found in all the compounds of the pyran-2-one cluster. It appeared then that most of the pyran-2-one compounds could not be clearly annotated due to the possibilities of many regio- or stereoisomerism. In this way, none of the three $\mathrm{m} / z$ 229.1047, 229.1048, 229.1049 nodes could be assigned as one of the four diastereoisomers of LL-P880 $\gamma$, among which only the $\left(1^{\prime} S, 2^{\prime} R\right)$ and $\left(1^{\prime} R, 2^{\prime} S\right)$ analogues have been described as natural products. It was the same for the $m / z 231.1237$ and 231.1225 nodes, for which two of the eight possible 2'-hydroxypestalotin (LL-P880 $\beta$ ) configurations have been described as natural products, these two in the $6 S$ series. Thus, as some of the compounds would likely be new ones, it was decided to engage the purification and structure elucidation of all pyran-2-one derivatives found in the MES-SSW extract.

\subsection{Isolation and Structure Elucidation of Pyran-2-ones}

Targeted isolation of pyran-2-ones was both MS- and UV-guided, focusing on compounds detected in the cluster (III) of the MN and other compounds, showing a UV spectrum typical of a pyrone system. This led to the isolation of 12 pyran-2-ones including five new fungal compounds (Figure 5). Six of the seven known compounds were identified as $\left(1^{\prime} R, 2^{\prime} S\right)$-LL-P880 $(5), 5,6$-dihydro-4-methoxy-2H-pyran-2-one (8) [45], (6S, $\left.1^{\prime} S, 2^{\prime} R\right)$ LL-P880 $\beta$ (9) [44], (6S,1'S)-pestalotin (10) [58,59], 1'R-dehydropestalotin (11) [60,61] and 6-pentyl-4-methoxy-2H-pyran-2-one (12) [61]. The identification of these substances was established by comparison of their physical and spectroscopic data with those reported previously and their absolute configuration was established by circular dichroism and comparison of their spectra with published data.

Compound 1 was obtained as a white amorphous powder. Its molecular formula was deduced as $\mathrm{C}_{7} \mathrm{H}_{10} \mathrm{O}_{4}$ with three degrees of unsaturation from the (+)-HRESIMS ions at $m / z$ 159.0652 $[\mathrm{M}+\mathrm{H}]^{+}$. The ${ }^{13} \mathrm{C}$ NMR spectrum (Table 3 ) showed seven carbons including one oxygenated methyl group, one unsaturated methine, one oxygenated methine, one methylene, one oxygenated methylene, one unsaturated quaternary carbon and one carbonyle. The ${ }^{1} \mathrm{H}-\mathrm{NMR}$ spectrum (Table 3$)$ revealed the presence of one methoxy group at $\delta_{\mathrm{H}} 3.77(3 \mathrm{H}$, $\left.\mathrm{s}, \mathrm{O}-\mathrm{CH}_{3}\right)$, one methine olefinic proton at $\delta_{\mathrm{H}} 5.16(1 \mathrm{H}, \mathrm{dd}, J=1.5 \mathrm{~Hz}, \mathrm{H}-3)$, one oxygenated methine at $\delta_{\mathrm{H}} 4.51(1 \mathrm{H}, \mathrm{m}, \mathrm{H}-6)$, two methylene protons at $\delta_{\mathrm{H}} 2.8(1 \mathrm{H}, \mathrm{ddd}, J=1.5,12.5$, $16.7 \mathrm{~Hz}, \mathrm{H}-5 \mathrm{a})$ and $2.28(1 \mathrm{H}, \mathrm{dd}, J=3.8,17.2 \mathrm{~Hz}, \mathrm{H}-5 \mathrm{~b})$ and two oxygenated methylene protons at $\delta_{\mathrm{H}} 3.73\left(1 \mathrm{H}, \mathrm{dd}, J=4.6,12.2 \mathrm{~Hz}, \mathrm{H}-1^{\prime} \mathrm{b}\right) ; 3.92\left(1 \mathrm{H}, \mathrm{m}, \mathrm{H}-1^{\prime} \mathrm{a}\right)$. Comparison of these data with those of 5,6-dihydro-(6R)-hydroxymethyl-4-methoxy-2H-pyran-2-one [62] revealed that the two compounds possessed the same planar structure. This was supported 
by ${ }^{1} \mathrm{H}-{ }^{1} \mathrm{H}$ COSY correlations between $\mathrm{H}-6\left(\delta_{\mathrm{H}} 4.51\right)$ and $\mathrm{H}-5 \mathrm{a} / \mathrm{H}-5 \mathrm{~b} / \mathrm{H}-1^{\prime} \mathrm{a} / \mathrm{H}-1^{\prime} \mathrm{b}$. HMBC correlations from $\mathrm{H}-3\left(\delta_{\mathrm{H}} 5.16\right)$ to $\mathrm{C}-2\left(\delta_{\mathrm{C}} 166.73\right) / \mathrm{C}-4\left(\delta_{\mathrm{C}} 172.94\right) / \mathrm{C}-5\left(\delta_{\mathrm{C}} 28.84\right)$, from H-5a $\left(\delta_{\mathrm{H}} 2.8\right)$ to $\mathrm{C}-3\left(\delta_{\mathrm{C}} 89.95\right) / \mathrm{C}-4\left(\delta_{\mathrm{C}} 174.94\right) / \mathrm{C}-6\left(\delta_{\mathrm{C}} 76.21\right) / \mathrm{C}-1^{\prime}\left(\delta_{\mathrm{C}} 63.74\right)$ and $4-\mathrm{OCH}_{3}$ to $\mathrm{C}-3$ $\left(\delta_{C} 89.95\right) / C-4\left(\delta_{C} 174.94\right)$ confirmed that the methoxy group and the hydroxymethyl were located at $C-4\left(\delta_{C} 174.94\right)$ and $C-6\left(\delta_{C} 76.21\right)$, respectively, of a 5,6-dihydro-2H-pyran-2-one moiety (Figure 6). The UV spectrum with an absorption band at $\lambda_{\max } 238 \mathrm{~nm}$ was also in accordance with a 5,6-dihydropyran-2-one moiety [43].<smiles>COC1=CC(=O)O[C@@H](CO)C1</smiles>

1<smiles>CCC[C@H](O)[C@H](O)[C@H]1CC(OC)=CC(=O)O1</smiles>

2: $\left(1^{\prime} S, 2^{\prime} S\right)$

3: $\left(1^{\prime} R, 2^{\prime} S\right)$<smiles>C/C=C/[C@H](O)[C@@H](O)[C@H]1CC(OC)=CC(=O)O1</smiles>

4<smiles>CCC[C@H](O)[C@H](O)c1cc(OC)cc(=O)o1</smiles>

5<smiles>C/C=C/[C@H](O)[C@@H](O)c1cc(OC)cc(=O)o1</smiles>

6<smiles>COc1ccoc(=O)c1</smiles>

7<smiles>COC1=CC(=O)OCC1</smiles>

8<smiles>CCC[C@H](O)[C@@H](O)[C@H]1CC(OC)=CC(=O)O1</smiles>

9<smiles>CCCC[C@H](O)[C@H]1CC(OC)=CC(=O)O1</smiles>

10<smiles>CCCC[C@H](O)c1cc(OC)cc(=O)o1</smiles>

11<smiles>CCCCCc1cc(OC)cc(=O)o1</smiles>

12

Figure 5. Structures of compounds 1-12.<smiles>O=C1C=CCC2(CCOCC2)CC1CO</smiles>

(1)<smiles>C/C=C/C(O)(CC)C1(O)CC(OC)CC(=O)O1</smiles>

(4)

$$
\overbrace{\text { HMBC }}^{\text {cosY }}
$$

Figure 6. Selected HMBC and COSY correlations for compound 1, 4.

The absolute configuration of 1 was assigned as $(6 S)$ using optical rotation which was found to be of a similar magnitude to that of 5,6-dihydro-(6R)-hydroxymethyl-4methoxy-2H-pyran-2-one [62], but opposite. The ECD spectrum confirmed this assignment as it showed a negative Cotton effect at $245 \mathrm{~nm}$ (Figure 7), similar to the one reported for $\left(6 S, 1^{\prime} S\right)$-pestalotin [59] and compound 9 (also isolated and analysed in this study). 5,6-dihydro-6-hydroxymethyl-4-methoxy-2H-pyran-2-one can be found, reported as two different products in the chemical literature. It is referenced under the CAS no: 1013918-20-1 as the (6R) enantiomer isolated from the endophytic fungus Pestalotiopsis sydowian and obtained as a synthetic intermediate by Pospisil et al., 2008 [63]. However, 
it is also found under the reference CAS no: $1332747-99-5$ as a compound isolated from the endophytic fungus Pestalotiopsis palmarum but without determination of its absolute configuration [62]. Therefore, compound $\mathbf{1}$ was assigned as the previously unreported 5,6-dihydro-6S-hydroxymethyl-4-methoxy-2H-pyran-2-one.

Table 3. ${ }^{1} \mathrm{H}(500 \mathrm{MHz})$ and ${ }^{13} \mathrm{C}$ NMR $(125 \mathrm{MHz})$ data for compounds 1-7 and the reference compound (6S,1'S,2'R)-LL-P880 $\beta$ (in $\mathrm{CDCl}_{3}$ ).

\begin{tabular}{|c|c|c|c|c|c|c|c|c|}
\hline \multirow{2}{*}{ Position } & \multicolumn{2}{|c|}{1} & \multicolumn{2}{|c|}{$\left(6 S, 1^{\prime} S, 2^{\prime} R\right)$-LL-P880 $\beta$} & \multicolumn{2}{|c|}{2} & \multicolumn{2}{|c|}{3} \\
\hline & $\delta_{C}$, type & $\delta_{H}(J$ in $\mathrm{Hz})$ & $\delta_{C}$, type & $\delta_{\mathrm{H}}(J$ in $\mathrm{Hz})$ & $\delta_{C}$, type & $\delta_{\mathrm{H}}(J$ in $\mathrm{Hz})$ & $\delta_{C}$, type & $\delta_{H}(J$ in $\mathrm{Hz})$ \\
\hline 2 & $166.73 \mathrm{C}$ & - & $166.58 \mathrm{C}$ & & $167.03 \mathrm{C}$ & - & $167.18 \mathrm{C}$ & - \\
\hline 3 & $89.95 \mathrm{CH}$ & $5.16, \mathrm{~s}$ & $89.73 \mathrm{CH}$ & $5.14(\mathrm{~d}, 1.5)$ & $89.51 \mathrm{CH}$ & $5.12, \mathrm{~s}$ & $89.55 \mathrm{CH}$ & $5.11, \mathrm{~s}$ \\
\hline 4 & $172.94 \mathrm{C}$ & - & $173.40 \mathrm{C}$ & & $173.94 \mathrm{C}$ & - & $173.68 \mathrm{C}$ & - \\
\hline 5 & $28.84 \mathrm{CH}_{2}$ & $\begin{array}{c}\text { a. } 2.8(\mathrm{dd}, \\
12.8,16.7) \\
\text { b. } 2.28(\mathrm{dd}, \\
3.7,17.0)\end{array}$ & $29.35 \mathrm{CH}_{2}$ & $\begin{array}{c}\text { a. } 2.89(\mathrm{ddd}, \\
1.5,12.9,17.2) \\
\text { b. } 2.32(\mathrm{dd}, \\
3.7,16.9)\end{array}$ & $29.49 \mathrm{CH}_{2}$ & $\begin{array}{c}\text { a. } 3.05(\mathrm{dd}, \\
13.8,16.7) \\
\text { b. } 2.19(\mathrm{dd}, \\
2.9,17.0)\end{array}$ & $29.30 \mathrm{CH}_{2}$ & $\begin{array}{c}\text { a. } 2.88(\mathrm{dd}, \\
13.9,16.2) \\
\text { b. } 2.31(\mathrm{dd}, \\
3.5,17.2)\end{array}$ \\
\hline 6 & $76.21 \mathrm{CH}$ & $4.51, \mathrm{~m}$ & $77.95 \mathrm{CH}$ & $\begin{array}{c}4.52(\mathrm{dt}, 4.0 \\
4.0,12.9)\end{array}$ & $75.41 \mathrm{CH}$ & $\begin{array}{c}4.76(\mathrm{dt}, 12.8 \\
2.3)\end{array}$ & $77.88 \mathrm{CH}$ & $\begin{array}{c}4.5(\mathrm{dt}, 3.9, \\
4.2,12.8)\end{array}$ \\
\hline $4-\mathrm{OCH}_{3}$ & 56.18 & $3.77, \mathrm{~s}$ & 56.16 & $3.76, \mathrm{~s}$ & 56.16 & $3.76, \mathrm{~s}$ & 56.18 & $3.74, \mathrm{~s}$ \\
\hline $1^{\prime}$ & $63.74 \mathrm{CH}_{2}$ & $\begin{array}{c}\text { a. } 3.73(\mathrm{dd}, \\
4.6,12.2) \\
\text { b. } 3.91, \mathrm{~m}\end{array}$ & $73.80 \mathrm{CH}$ & $\begin{array}{c}3.49(\mathrm{dd}, 2.9 \\
4.2)\end{array}$ & $71.27 \mathrm{CH}$ & $\begin{array}{l}3.37 \text { (dd, } 2.0 \\
6.7)\end{array}$ & $70.77 \mathrm{CH}$ & $\begin{array}{c}3.47(\mathrm{t}, 3.5, \\
3.9)\end{array}$ \\
\hline $2^{\prime}$ & - & - & $70.93 \mathrm{CH}$ & $3.80, \mathrm{~m}$ & $74.13 \mathrm{CH}$ & $\begin{array}{c}3.80(\mathrm{t}, 7.1 \\
7.7)\end{array}$ & $73.78 \mathrm{CH}$ & $\begin{array}{c}3.77(\mathrm{t}, 3.5, \\
4.3)\end{array}$ \\
\hline $3^{\prime}$ & - & - & $35.94 \mathrm{CH}_{2}$ & $1.5-1.63, \mathrm{~m}$ & $35.71 \mathrm{CH}_{2}$ & $\begin{array}{l}\text { a. } 1.66, \mathrm{~m} \\
\text { b. } 1.5, \mathrm{~m}\end{array}$ & $35.84 \mathrm{CH}_{2}$ & $1.5-1.58, \mathrm{~m}$ \\
\hline $4^{\prime}$ & - & - & $18.78 \mathrm{CH}_{2}$ & $1.38-1.5, \mathrm{~m}$ & $18.78 \mathrm{CH}_{2}$ & $\begin{array}{l}\text { a. } 1.58, \mathrm{~m} \\
\text { b. } 1.4, \mathrm{~m}\end{array}$ & $18.72 \mathrm{CH}_{2}$ & $1.38-1.5, \mathrm{~m}$ \\
\hline $5^{\prime}$ & - & - & $13.94 \mathrm{CH}_{3}$ & $0.94(t, 7.1)$ & $13.98 \mathrm{CH}_{3}$ & $0.95(t, 7.1)$ & $13.92 \mathrm{CH}_{3}$ & $0.92(\mathrm{t}, 6.7)$ \\
\hline \multirow{2}{*}{ Position } & \multicolumn{2}{|c|}{4} & \multicolumn{2}{|c|}{5} & \multicolumn{2}{|c|}{6} & \multicolumn{2}{|c|}{7} \\
\hline & $\delta_{C}$, type & $\delta_{\mathrm{H}}(J$ in $\mathrm{Hz})$ & $\delta_{C}$, type & $\delta_{H}(J$ in $\mathrm{Hz})$ & $\delta_{C}$, type & $\delta_{\mathrm{H}}(J$ in $\mathrm{Hz})$ & $\delta_{C}$, type & $\delta_{H}(J$ in $\mathrm{Hz})$ \\
\hline 2 & $166.58 \mathrm{C}$ & & $164.12 \mathrm{C}$ & & $162.7 \mathrm{C}$ & & $163.75 \mathrm{C}$ & \\
\hline 3 & $89.81 \mathrm{CH}$ & $5.14, \mathrm{~s}$ & $88.4 \mathrm{CH}$ & $5.46(\mathrm{~d}, 2.0)$ & $88.35 \mathrm{CH}$ & $5.44(\mathrm{~d}, 2.7)$ & $89.98 \mathrm{CH}$ & $5.53(\mathrm{~d}, 2.3)$ \\
\hline 4 & $173.32 \mathrm{C}$ & & $171.11 \mathrm{C}$ & & $171.18 \mathrm{C}$ & & $170.56 \mathrm{C}$ & \\
\hline 5 & $29.55 \mathrm{CH}_{2}$ & $\begin{array}{c}\text { a. } 2.97(\mathrm{dd}, \\
13.8,17.3) \\
\text { b. } 2.27(\mathrm{dd}, \\
3.9,17.3)\end{array}$ & $100.45 \mathrm{CH}$ & $6.16(\mathrm{~d}, 2.0)$ & $100.19 \mathrm{CH}$ & $6.14(\mathrm{~d}, 2.0)$ & $103.13 \mathrm{CH}$ & $6.01, \mathrm{~m}$ \\
\hline 6 & $75.94 \mathrm{CH}$ & $\begin{array}{c}4.51(\mathrm{dt}, 3.2 \\
3.2,12.8)\end{array}$ & $163.19 \mathrm{C}$ & - & $167.3 \mathrm{C}$ & - & $150.90 \mathrm{CH}$ & $7.35(\mathrm{~d}, 6.1)$ \\
\hline $4-\mathrm{OCH}_{3}$ & 56.18 & $3.76, \mathrm{~s}$ & 55.99 & $3.83, \mathrm{~s}$ & 55.96 & $3.82, \mathrm{~s}$ & 55.51 & $3.81, \mathrm{~s}$ \\
\hline $1^{\prime}$ & $74.79 \mathrm{CH}$ & $\begin{array}{c}3.49(\mathrm{dd}, 2.0 \\
6.1)\end{array}$ & $73.44 \mathrm{CH}$ & $4.44(\mathrm{~d}, 4.0)$ & $73.75 \mathrm{CH}$ & $4.51, \mathrm{br}, \mathrm{m}$ & - & - \\
\hline $2^{\prime}$ & $72.62 \mathrm{CH}$ & $4.32(t, 6.4)$ & $72.56 \mathrm{CH}$ & $4.02, \mathrm{~m}$ & $72.98 \mathrm{CH}$ & $4.51, \mathrm{br}, \mathrm{m}$ & - & - \\
\hline $3^{\prime}$ & $129.24 \mathrm{CH}$ & $\begin{array}{c}5.53(\mathrm{dd}, 7.7 \\
15.7)\end{array}$ & $33.24 \mathrm{CH}_{2}$ & $1.43, \mathrm{~m}$ & $127.1 \mathrm{CH}$ & $\begin{array}{c}5.5(\mathrm{dd}, 1.3 \\
2.0,15.5)\end{array}$ & - & - \\
\hline $4^{\prime}$ & $130.60 \mathrm{CH}$ & $5.88, \mathrm{~m}$ & $18.91 \mathrm{CH}_{2}$ & $\begin{array}{l}\text { a. } 1.54, \mathrm{~m} \\
\text { b. } 1.35, \mathrm{~m}\end{array}$ & $131.48 \mathrm{CH}$ & $\begin{array}{c}5.83(\mathrm{dq}, 6.7 \\
15.5)\end{array}$ & - & - \\
\hline $5^{\prime}$ & $17.88 \mathrm{CH}_{3}$ & $1.74(\mathrm{~d}, 6.4)$ & $13.91 \mathrm{CH}_{3}$ & $0.93(t, 7.4)$ & $17.9 \mathrm{CH}_{3}$ & $\begin{array}{c}1.71(\mathrm{dd}, 1.3 \\
6.7)\end{array}$ & - & - \\
\hline
\end{tabular}

Compound 2 was obtained as a white powder. Its (+)-HRESIMS data $(m / z 231.1229$ $[\mathrm{M}+\mathrm{H}]^{+}, 253.1050[\mathrm{M}+\mathrm{Na}]^{+}$) indicated a molecular formula of $\mathrm{C}_{11} \mathrm{H}_{18} \mathrm{O}_{5}$ corresponding to a hydroxylated derivative of pestalotin such as $\left(6 S, 1^{\prime} S, 2^{\prime} R\right)$-LL-P880 $\beta$ [44]. The UV spectrum in $\mathrm{MeOH}$ showed an absorption maximum at $238 \mathrm{~nm}$, revealing a 5,6-dihydropyran2-one moiety [43]. The ${ }^{13} \mathrm{C}$ NMR spectrum displayed 11 carbons including two methyl groups, three methylenes, three methines, and two quaternary carbons. Inspection of the ${ }^{1} \mathrm{H}$ NMR spectrum showed one methyl group at $\delta_{\mathrm{H}} 0.95\left(3 \mathrm{H}, \mathrm{t}, J=7.1 \mathrm{~Hz}, \mathrm{H}-5^{\prime}\right)$, one olefinic proton at $\delta_{\mathrm{H}} 5.12(1 \mathrm{H}, \mathrm{s}, \mathrm{H}-3)$, six methylenes at $\delta_{\mathrm{H}} 1.38-1.5\left(2 \mathrm{H}, \mathrm{m}, \mathrm{H}-4^{\prime}\right), \delta_{\mathrm{H}}$ $1.5-1.63\left(2 \mathrm{H}, \mathrm{m}, \mathrm{H}-3^{\prime}\right), \delta_{\mathrm{H}} 3.05(1 \mathrm{H}, \mathrm{dd}, J=13.8,16.7 \mathrm{~Hz}, \mathrm{H}-5 \mathrm{a}), \delta_{\mathrm{H}} 2.19(1 \mathrm{H}, \mathrm{dd}, J=2.9$, $17.0 \mathrm{~Hz}, \mathrm{H}-5 \mathrm{~b})$, three oxygenated methines $\delta_{\mathrm{H}} 3.37\left(1 \mathrm{H}, \mathrm{dd}, J=2.0,6.7 \mathrm{~Hz}, \mathrm{H}-1^{\prime}\right), \delta_{\mathrm{H}} 3.80$ $\left(1 \mathrm{H}, \mathrm{t}, J=7.1,7.7 \mathrm{~Hz}, \mathrm{H}-2^{\prime}\right), \delta_{\mathrm{H}} 4.76(1 \mathrm{H}, \mathrm{d}, J=12.8 \mathrm{~Hz}, \mathrm{H}-6)$ and one methoxy group $\delta_{\mathrm{H}}$ 
$3.76\left(3 \mathrm{H}, \mathrm{s}, 4-\mathrm{OCH}_{3}\right)$. The analysis of the ${ }^{1} \mathrm{H}_{-}{ }^{1} \mathrm{H}$ COSY spectrum indicated the presence of a spin system (C-3-C-5-C-6-C-1'-C-2'-C-3'-C-4'-C-5'). Key HMBC correlations from H-3 $\left(\delta_{\mathrm{H}} 5.12\right)$ to $\mathrm{C}-2\left(\delta_{\mathrm{C}} 167.03\right) / \mathrm{C}-4\left(\delta_{\mathrm{C}} 173.94\right) / \mathrm{C}-5\left(\delta_{\mathrm{C}} 29.49\right) / \mathrm{C}-6\left(\delta_{\mathrm{C}} 75.41\right)$, from H-1' $\left(\delta_{\mathrm{H}}\right.$ $3.37)$ to $\mathrm{C}-2\left(\delta_{\mathrm{C}} 167.03\right) / \mathrm{C}-5\left(\delta_{\mathrm{C}} 29.49\right) / \mathrm{C}-6\left(\delta_{\mathrm{C}} 75.41\right) / \mathrm{C}-2^{\prime}\left(\delta_{\mathrm{C}} 74.13\right), \mathrm{H}-5 \mathrm{a}\left(\delta_{\mathrm{H}} 3.05\right)$ to $\mathrm{C}-2\left(\delta_{\mathrm{C}} 167.03\right) / \mathrm{C}-3\left(\delta_{\mathrm{C}} 89.51\right) / \mathrm{C}-4\left(\delta_{\mathrm{C}} 173.94\right) / \mathrm{C}-6\left(\delta_{\mathrm{C}} 75.41\right) / \mathrm{C}-1^{\prime}\left(\delta_{\mathrm{C}} 71.27\right)$ and from $4-\mathrm{OCH}_{3}\left(\delta_{\mathrm{H}} 3.76\right)$ to $\mathrm{C}-2\left(\delta_{\mathrm{C}} 167.03\right) / \mathrm{C}-4\left(\delta_{\mathrm{C}} 173.94\right) / \mathrm{C}-5\left(\delta_{\mathrm{C}} 29.49\right)$ confirmed that the methoxy group and an $1^{\prime}, 2^{\prime}$-dihydroxy five-carbon side chain were, respectively, located at C-4 and C- 6 of the 5,6-dihydro-pyran-2-one. The planar structure of $\mathbf{2}$ was then established as the same as hydroxypestalotin $\left[\left(6 S, 1^{\prime} S, 2^{\prime} R\right)\right.$-LL-P880 $\left.\beta\right]$. The ECD spectrum of compound 2 was similar to compound $1\left(\Delta \varepsilon_{240.9} \mathrm{~nm}=-1.92\right)$ and $\left(6 S, 1^{\prime} S, 2^{\prime} R\right)$-LL-P880 $\beta$ $\left(\Delta \varepsilon_{245.2 \mathrm{~nm}}=-11.54\right)$ with a negative Cotton effect at $247 \mathrm{~nm}$ indicating its (6S) absolute configuration (Figure 7). The four diastereoisomers SSR, SSS, SRS and SRR have been reported so far, but only the two first have been isolated from a natural source. Their absolute configuration has been, for most of them, deduced from diagnostic coupling constants of the $\mathrm{H}-6-\mathrm{H}-1^{\prime}-\mathrm{H} 2^{\prime}$ system, which we investigated in the ${ }^{1} \mathrm{H}$ NMR spectrum of 2. The ${ }^{3} J_{\mathrm{H}-6-\mathrm{H}-1^{\prime}}(J=2.0 \mathrm{~Hz})$ of 2 was different to that of $\left(6 S, 1^{\prime} R, 2^{\prime} S\right)$-nodulisporipyrone $\mathrm{D}$ $\left({ }^{3} J_{\mathrm{H}-6-\mathrm{H}-1^{\prime}}=6.5 \mathrm{~Hz}\right)[64]$ and $\left(6 R, 1^{\prime} S, 2^{\prime} R\right)$-LL-P880 $\beta\left({ }^{3} J_{\mathrm{H}-6-\mathrm{H}-1^{\prime}}=6.7 \mathrm{~Hz}\right)[65]$ but similar to that of $\left(6 S, 1^{\prime} S, 2^{\prime} S\right)-\mathrm{LL}-\mathrm{P} 880 \beta\left({ }^{3} J_{\mathrm{H}-6-\mathrm{H}-1^{\prime}}=1.1 \mathrm{~Hz}\right)[66]$, which indicated that the relationship between $\mathrm{H}-6$ and $\mathrm{H}-1^{\prime}$ was syn. Moreover, the ${ }^{3} J_{\mathrm{H}-1^{\prime}-\mathrm{H}-2^{\prime}}(J=6,7 \mathrm{~Hz})$ of 2 was equivalent to that of $\left(6 S, 1^{\prime} R, 2^{\prime} R\right)$-LL-P880 $\beta\left({ }^{3} J_{\mathrm{H}-1^{\prime}-\mathrm{H}-2^{\prime}}=6.1 \mathrm{~Hz}\right)[67]$ and in the same order as that of $\left(6 R, 1^{\prime} R, 2^{\prime} R\right)$-LL-P880 $\beta\left({ }^{3} J_{\mathrm{H}-1^{\prime}-\mathrm{H}-2^{\prime}}=9.2 \mathrm{~Hz}\right)[67]$ and $\left(6 S, 1^{\prime} S, 2^{\prime} S\right)$-LL-P880 $\beta$ $\left({ }^{3} J_{\mathrm{H}-1^{\prime}-\mathrm{H}-2^{\prime}}=9.1 \mathrm{~Hz}\right)[66]$, which identified that the relationship between $\mathrm{H}-\mathrm{1}^{\prime}$ and $\mathrm{H}-\mathrm{2}^{\prime}$ was anti. Furthermore, the NOESY spectrum showed both an absence of correlation between $\mathrm{H}-6$ and $\mathrm{H}-2^{\prime}$ and a strong correlation between $\mathrm{H}-1^{\prime}$ and $\mathrm{H}-2^{\prime}$. On the basis on these results, the absolute configuration of 2 was determined as $6 S, 1^{\prime} S, 2^{\prime} S$ (Figure 8) and compound 2 was assigned as $\left(6 S, 1^{\prime} S, 2^{\prime} S\right)$-LL-P880 $\beta$, a natural product obtained once from a mangroved-derived Pestalotiopsis virgatula [66].
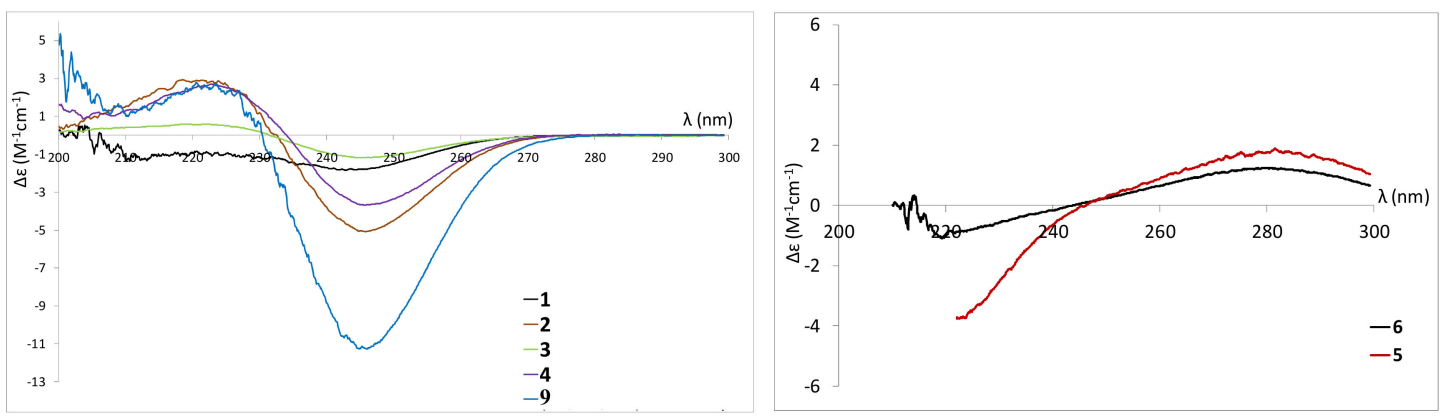

Figure 7. ECD spectra of compounds 1-6 and reference compound $\mathbf{9}$ monitored in methanol.<smiles></smiles>

(2)

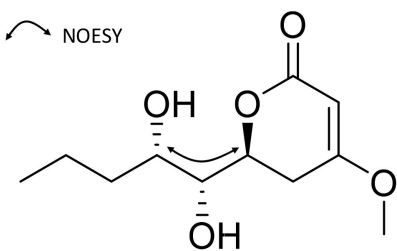

(3)<smiles>CCC[C@H](O)[C@H](O)C[C@@H]1CC(OC)=CC(=O)O1</smiles>

(9)

Figure 8. Selected NOESY correlations for compounds 2, 3 and $\mathbf{9 .}$

Compound 3 was obtained as a white powder and showed the same molecular formula $\mathrm{C}_{11} \mathrm{H}_{18} \mathrm{O}_{5}$ as compound 2, with ions $m / z 231.1225[\mathrm{M}+\mathrm{H}]^{+}, 253.1041[\mathrm{M}+\mathrm{Na}]^{+}$in (+)-HRESIMS. The 1D NMR ( ${ }^{1} \mathrm{H},{ }^{13} \mathrm{C}$ NMR), 2D NMR (HSQC, COSY, and HMBC), UV and ECD spectra were very similar to those of 2 , indicating the same planar structure. However, the ${ }^{3} J_{\mathrm{H} 6-\mathrm{H} 1^{\prime}}(J=3.9 \mathrm{~Hz})$ was different from 2 but similar to $\left(6 S, 1^{\prime} R, 2^{\prime} R\right)$-LL-P880 $\beta$, 
$\left({ }^{3} J_{\mathrm{H} 6-\mathrm{H} 1^{\prime}}=4.0 \mathrm{~Hz}\right)[67]$, and ${ }^{3} J_{\mathrm{H}-1^{\prime}-\mathrm{H}-2^{\prime}}(J=3.5 \mathrm{~Hz})$ of 3 was different from the one of 2 but

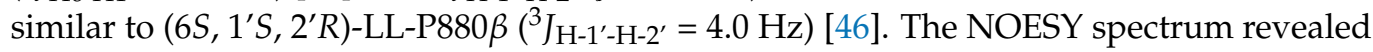
a strong H-6/H-2' correlation and the absence of $\mathrm{H}-\mathbf{1}^{\prime} / \mathrm{H}-2^{\prime}$, and $\mathrm{H}-\mathrm{1}^{\prime} / \mathrm{H}-6$ correlation (Figure 8). Compound 3 was then assigned as $\left(6 S, 1^{\prime} R, 2^{\prime} S\right)$-LL-P880 $\beta$, a new natural product previously obtained by stereoselective synthesis [68].

Compound 4 was isolated as a white amorphous powder. Its (+)-HRESIMS spectrum displayed ions at $m / z 229.1068[\mathrm{M}+\mathrm{H}]^{+}, 251.0884[\mathrm{M}+\mathrm{Na}]^{+}$, and $211.0964\left[\mathrm{M}-\mathrm{H}_{2} \mathrm{O}+\mathrm{H}\right]^{+}$, with the formula $\mathrm{C}_{11} \mathrm{H}_{16} \mathrm{O}_{5}$ and four degrees of unsaturation. The UV spectrum in $\mathrm{MeOH}$ showed an absorption maximum at $238 \mathrm{~nm}$, revealing a 5,6-dihydro-pyran-2-one moiety [43]. The ${ }^{1} \mathrm{H}-\mathrm{NMR}$ and ${ }^{13} \mathrm{C}-\mathrm{NMR}$ spectra have demonstrated that 4 was closely related with 2 and 3 except for the difference in the chemical shift of $\mathrm{H}-2^{\prime}\left(\delta_{\mathrm{H}} 4.32\right) / \mathrm{C}-2^{\prime}\left(\delta_{\mathrm{H}} 72.62\right)$, $\mathrm{H}-3^{\prime}\left(\delta_{\mathrm{H}} 5.53\right) / \mathrm{C}-3^{\prime}\left(\delta_{\mathrm{C}} 129.24\right), \mathrm{H}-4^{\prime}\left(\delta_{\mathrm{H}} 5.88\right) / \mathrm{C}-4^{\prime}\left(\delta_{\mathrm{C}} 130.60\right)$ and $\mathrm{H}-5^{\prime}\left(\delta_{\mathrm{H}} 1.74\right) / \mathrm{C}-5^{\prime}\left(\delta_{\mathrm{C}}\right.$ 17.88). The differences in molecular formula and NMR data indicated that 4 was a reduced homologue of 2, 3 and 9. The HMBC correlation from H-4' $\left(\delta_{\mathrm{H}} 5.88\right)$ to $\mathrm{C}-2^{\prime}\left(\delta_{\mathrm{C}}\right.$ $72.62) / \mathrm{C}-5^{\prime}\left(\delta_{\mathrm{C}} 17.88\right)$ and from $\mathrm{H}-2^{\prime}\left(\delta_{\mathrm{H}} 4.32\right)$ to $\mathrm{C}-1^{\prime}\left(\delta_{\mathrm{C}} 74.79\right) / \mathrm{C}-3^{\prime}\left(\delta_{\mathrm{C}} 129.24\right) / \mathrm{C}-4^{\prime}\left(\delta_{\mathrm{C}}\right.$ $130.60) / C-6\left(\delta_{C} 75.94\right)$ indicated that the double bond was adjacent to a methyl group and occurred at $C-3^{\prime}$ and $C-4^{\prime}$. The geometry of $C-3^{\prime}\left(\delta_{C} 129.24\right)$ was deduced to be $E$ by the NOESY correlation between $\mathrm{H}-2^{\prime}$ and $\mathrm{H}-4^{\prime}$, the ${ }^{13} \mathrm{C}-\mathrm{NMR}$ deshielded chemical shift of the methyl group and the coupling constant ${ }^{3} \mathrm{H}_{\mathrm{H}^{\prime}-\mathrm{H} 4^{\prime}}=15.7 \mathrm{~Hz}$. The planar structure of 4 was established, which was confirmed by the exhaustive analysis of the 2D-NMR data (HSQC, ${ }^{1} \mathrm{H}-{ }^{1} \mathrm{H}$ COSY, and HMBC) (Figure 6). The ECD spectrum of compound 4 was found to be similar to those of 1-3 (Figure 7). NOESY correlations between H-6 and H-1' and between $\mathrm{H}-1^{\prime}$ and $\mathrm{H}-2^{\prime}$ together with diagnostic coupling constants ${ }^{3} \mathrm{~J}_{\mathrm{H} 6-\mathrm{H} 1^{\prime}}=2.0 \mathrm{~Hz}$ (similar to that of $\left(6 S, 1^{\prime} S, 2^{\prime} S\right)$-LL-P880 $\beta[66]$ and ${ }^{3} J_{\mathrm{H} 1^{\prime}-\mathrm{H} 2^{\prime}}=6.1 \mathrm{~Hz}$ (equivalent to that of 2), permitted the assignment of the structure and absolute configuration for 4 as the new compound 5,6-dihydro-4-methoxy-6S-(1'S,2'S-dihydroxy pent-3'(E)-enyl)-2H-pyran-2-one, unprecedented LL-P880 $\beta$ analogue with an unsaturated side chain.

Compound 5 was obtained as a white amorphous powder. The (+)-HRESIMS analysis returned a molecular formula of $\mathrm{C}_{11} \mathrm{H}_{16} \mathrm{O}_{5}$ with four degrees of unsaturation from ions $m / z 229.1069[\mathrm{M}+\mathrm{H}]^{+}, 251.0896[\mathrm{M}+\mathrm{Na}]^{+}$, and $211.0969\left[\mathrm{M}-\mathrm{H}_{2} \mathrm{O}+\mathrm{H}\right]^{+}$. The UV spectrum of compound 5 in $\mathrm{MeOH}$ showed an absorption maximum at $280 \mathrm{~nm}$ characteristic of an unsaturated pyran-2-one moiety [42]. The ${ }^{1} \mathrm{H}$-NMR spectrum showed the presence of one methoxy group at $\delta_{\mathrm{H}} 3.83\left(3 \mathrm{H}, \mathrm{s}, 4-\mathrm{OCH}_{3}\right)$, a pair of olefinic hydrogens at $\delta_{\mathrm{H}} 5.46(1 \mathrm{H}, \mathrm{d}$, $J=2.0 \mathrm{~Hz}, \mathrm{H}-3)$ and $6.16(1 \mathrm{H}, \mathrm{d}, J=2.0 \mathrm{~Hz}, \mathrm{H}-5)$, a methyl at $\delta_{\mathrm{H}} 0.93\left(3 \mathrm{H}, 7.1, \mathrm{H}-5^{\prime}\right)$, four methylenes at $\delta_{\mathrm{H}} 1.43\left(2 \mathrm{H}, \mathrm{m}, \mathrm{H}-3^{\prime}\right), \delta_{\mathrm{H}} 1.54\left(1 \mathrm{H}, \mathrm{m}, \mathrm{H}-4^{\prime}\right)$ and $\delta_{\mathrm{H}} 1.35\left(1 \mathrm{H}, \mathrm{m}, \mathrm{H}-4^{\prime}\right)$, and two oxygenated $\mathrm{sp}^{3}$ methines at $\delta_{\mathrm{H}} 4.44\left(1 \mathrm{H}, \mathrm{d}, J=4.0 \mathrm{~Hz}, \mathrm{H}-1^{\prime}\right)$ and $4.02\left(1 \mathrm{H}, \mathrm{m}, \mathrm{H}-2^{\prime}\right)$. These data suggested that compound $\mathbf{5}$ was the reduced product of $\mathbf{3}$ in the lactone ring, which was confirmed by comparison of the ${ }^{13} \mathrm{C}, \mathrm{COSY}, \mathrm{HSQC}$ and HMBC spectra with those of LL-P880 $\gamma[44,46,69]$. The coupling constant ${ }^{3} J_{\mathrm{H}^{\prime}-\mathrm{H} 2^{\prime}}=4.0 \mathrm{~Hz}$ and a correlation observed between $\mathrm{H}-1^{\prime}$ and $\mathrm{H}-2^{\prime}$ in the NOESY spectrum experiment of 5 indicated that they were syn and that the relative configuration of 5 was $1^{\prime} R^{*}, 2^{\prime} S^{*}$ or $1^{\prime} S^{*}, 2^{\prime} R^{*}$. The optical rotation of compound 5, with $[\alpha]_{D}^{20}+63($ c $1.0, \mathrm{MeOH})$, had the same sign than $\left(1^{\prime} R, 2^{\prime} S\right)$-LL-P880 $\gamma$ and $\left(1^{\prime} R, 2^{\prime} R\right)$-LL-P880 $\gamma$ and the ECD spectrum of compound $5\left(\Delta \varepsilon_{281.9 n m}=+2.042\right)$ exhibited a positive Cotton effect at $282 \mathrm{~nm}$ already described for $6-\left(1 R, 2^{\prime} R\right.$-dihydroxyheptyl)-4methoxy-2H-pyran-2-one and 6-(1R, 2'S-dihydroxyheptyl)-4-methoxy-2H-pyran-2-one [64]. These observations allowed the assignment of the structure and absolute configuration of 5 as the new natural product $\left(1^{\prime} R, 2^{\prime} S\right)$-LL-P880 $\gamma$, previously described as a synthetic compound [69] and reported from Xylaria feejeenisis but without stereochemistry data [70].

Compound 6 was isolated as a white amorphous powder. The (+)-HRESIMS spectrum of 6 displayed ions at $\mathrm{m} / z 227.0921[\mathrm{M}+\mathrm{H}]^{+}, 249.0743[\mathrm{M}+\mathrm{Na}]^{+}$, and 209.0822 $\left[\mathrm{M}-\mathrm{H}_{2} \mathrm{O}+\mathrm{H}\right]^{+}$, corresponding to the formula $\mathrm{C}_{11} \mathrm{H}_{14} \mathrm{O}_{5}$ with five degrees of unsaturation. This difference in molecular formula compared to $5(-2 \mathrm{H})$ and its UV spectrum presenting the same $\lambda_{\max }=280 \mathrm{~nm}$ indicated that $\mathbf{6}$ was a reduction product of 5 on the side chain of the pyran-2-one moiety. The ${ }^{1} \mathrm{H}$ and ${ }^{13} \mathrm{C}-\mathrm{NMR}$ spectra (Table 3 ) were also 
very similar to those of compound 5, except for the absence of the signals corresponding to the $\mathrm{H}-3^{\prime}$ and $\mathrm{H}-4^{\prime}$ methylenes. Instead, two olefinic methines were observed at $\delta_{\mathrm{H}} 5.5$ $\left(1 \mathrm{H}, \mathrm{ddd}, J=2.01,1.3,15.5 \mathrm{~Hz}, \mathrm{H}-3^{\prime}\right)$ and $\delta_{\mathrm{H}} 5.83\left(1 \mathrm{H}, \mathrm{dq}, J=6.7,15.5 \mathrm{~Hz}, \mathrm{H}-4^{\prime}\right)$ with an $\mathrm{HMBC}$ correlation from $\mathrm{H}-5^{\prime}$ to $\mathrm{C}-3^{\prime}$ and $\mathrm{C}-4^{\prime}$ suggesting that the double bond was adjacent to the methyl group. A mutual transform coupling $(J=15.5 \mathrm{~Hz})$ indicated an $E$ configuration of the double bond. Based on the above analysis, the planar structure of $\mathbf{6}$ was established. The absolute configuration at $C-1^{\prime}$ was deduced to be $R$ from the observation that the ECD spectrum of compound $\mathbf{6}$ was similar to that of 5 (Figure 7). According to these data, the relative configuration of compound 6 could be proposed as $1^{\prime} R^{*}, 2^{\prime} R^{*}$ or $1^{\prime} R^{*}, 2^{\prime} S^{*}$. The ${ }^{1} \mathrm{H}$ and ${ }^{13} \mathrm{C}$ NMR shifts of these two possible stereoisomers were computed using density functional theory. After correlating the experimental and simulated data through the DP4 probability method [71,72], the relative configuration of $1^{\prime} R, 2^{\prime} R$ was identified as the most likely candidate in high probability, with $99.9 \%$ (both ${ }^{13} \mathrm{C}$ and ${ }^{1} \mathrm{H}$ NMR), 99.8\% (only ${ }^{1} \mathrm{H}$ NMR), and $73.9 \%$ (only ${ }^{13} \mathrm{C}$ NMR) probabilities values according to DP4 calculations (Figure 9). Finally, the structure of compound 6 was established as 4-methoxy-6-(1'R, 2'R-dihydroxy pent-3'(E)-enyl)-2H-pyran-2-one.

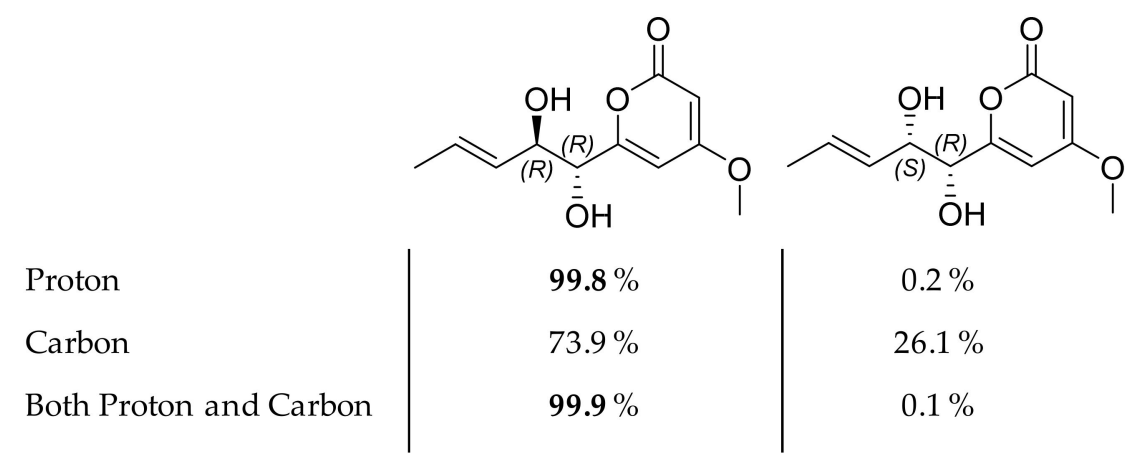

Figure 9. Chemical structures of two possible stereoisomers of compound 6 with their respective DP4 probabilities.

Compound 7 had the molecular formula $\mathrm{C}_{6} \mathrm{H}_{6} \mathrm{O}_{3}$ as indicated by the (+)-HRESIMS $m / z 127.0416[\mathrm{M}+\mathrm{H}]^{+}$. As for compounds 5 and $\mathbf{6}$, the UV spectrum of compound 7 was consistent with the presence of a pyran-2-one moiety [42]. The ${ }^{1} \mathrm{H},{ }^{13} \mathrm{C}-\mathrm{NMR}$ analysis (Table 3) allowed a final identification of compound 7 as 4-methoxy- $2 \mathrm{H}$-pyran-2-one. Compound 7 was originally reported as a synthetic intermediate during the synthesis of patulin [73] and, surprisingly, recently as a constituent of the roots of Glycyrrhiza uralensis [74]. It is described here for the first time as a fungal natural product.

\subsection{Biological Evaluation of Isolated Compounds}

Due to the limited amount, only some of the isolated products were tested for cytotoxic, antibacterial and antileishmanial activities. Compounds 7, 10-12 did not exhibit antiproliferative activity against the epithelial cancer cell line KB when assayed at a concentration of $50 \mu \mathrm{g} / \mathrm{mL}$. Compounds 2, 3, 7, 10-12 were screened for their antibacterial activities against Escherichia coli, Pseudomonas aeruginosa, Staphylococcus aureus and Enterococcus faecalis. None of them showed antibacterial activity at a concentration of $100 \mu \mathrm{g} / \mathrm{mL}$. Antileishmanial activity of $\mathbf{1 0}$ was evaluated through determination of $50 \%$ efficient concentration against Leishmania infantum amastigotes. The results of this assay showed no detectable activity at concentrations $\leq 50 \mu \mathrm{g} / \mathrm{mL}$.

\subsection{PTP1B Docking}

Recently, 3,4,6-trisubstituted pyran-2-one derivatives, chrysopyrones A and B have been described as potent inhibitors of protein tyrosine phosphatase 1B (PTP1B) [75]. To investigate a possible interaction between compound 10 and PTP1B, a molecular docking was performed using Gold software [76]. As shown in Figure 10, a docking result revealed 
that the carbonyl group of the pyran-2-one core could form hydrogen bonds with the -NH of Gly220 and -SH of Cys215, which is essential for catalysis [77,78], while the hydroxyl group could form a hydrogen bond with the carbonyl oxygen atom of the Gln262 amide side chain, another important residue in the second catalytic step [79]. The hydrophobic side of the molecule also makes van der Waals contacts with the side chains of Tyr46, Val49, Ala217 and Ile219. All these features are consistent with a possible inhibition of PTP1B that will have to be confirmed by experimental assays.

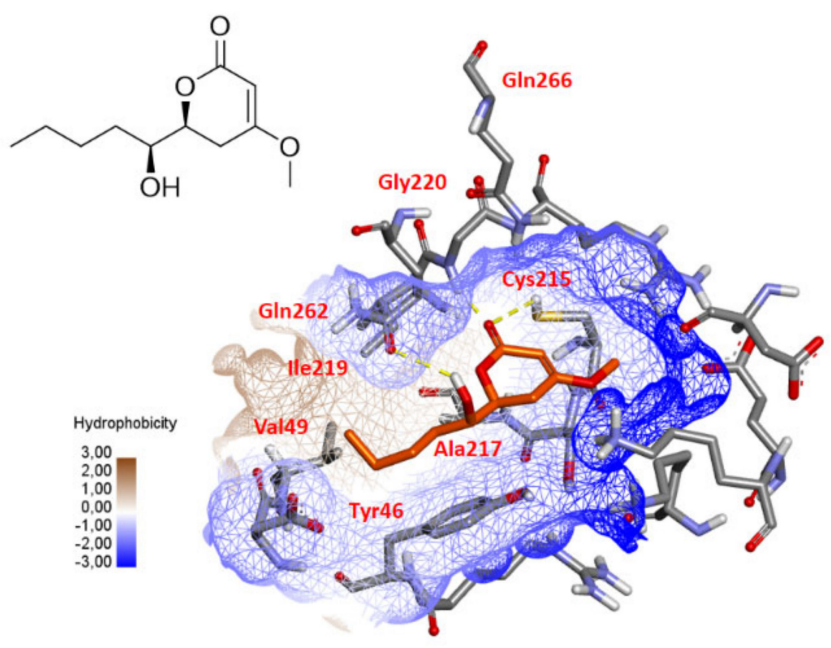

Figure 10. Possible docking solution of compound 10 (goldscore.fitness: 32.2589) in the active site of PTP1B (1NWL.pdb). Hydrogen bonds are indicated as yellow lines.

\section{Discussion}

Stress adaptation and metabolic response are critical mechanisms for the survival of microbes in a dynamic environment and for the colonization of host niches. Fungi can sense and then transduce external changes in osmotic pressure mainly through cellular signalling pathways such as the HOG pathway and then activated transcriptional activators will modulate the pattern of gene expression, leading to the production of osmoprotectant compounds such as glycerol [80]. Some studies in the literature have shown that the response to osmotic stress by fungi is in some cases linked to the biosynthesis of natural products [80-83]. For investigating new bioactive compounds in marine fungi, several strategies have been used to induce the expression of silent natural products including the use of abiotic stresses such as high $\mathrm{NaCl}$ concentrations [81,82]. In Aspergillus aculeatus, osmotic and saline stress exerted by glycerol and $\mathrm{NaCl}$, respectively, can modulate the production of natural products [82]. Similarly, the expression of polyketides was upregulated and de novo observed following the use of a combination of SAHA and osmotic stress in $A$. cruciatus [80]. Here, we observed that it was the combination of the use of seawater and the mussel-derived medium that induced the overexpression of some specialized compounds, i.e., culture conditions that can be considered as the closest to the original environment of the $P$. restrictum strain. This illustrates that there may be a specific metabolome of the living conditions within holobionts such as bivalves, which can be tentatively reconstituted in vitro.

The use of both metabolomics and feature-based molecular networking (FBMN) allowed to highlight compounds and to cluster them into chemical series. These tools are of high value for enhancing the dereplication of known compounds and can allow annotation propagation for the discovery of new analogs in a chemical series [84,85], as is shown here for melearorides. However, for small molecules and trace compounds such as the pyran-2-ones described here, fragmentation information is usually poor and they can escape MS/MS fragmentation because they are below the method threshold, particularly in complex mixtures such as fungal culture extracts. Furthermore, some compounds can 
be undetected and unrepresented in the $\mathrm{MN}$, as in the case of isomers, which can lead to similar fragmentation patterns. The observation of multiple isobaric compounds in an MN highlights these limits of MS-based dereplication, as shown in this study for LL-P880 $\beta$ stereoisomers. Then, adding further physico-chemical properties such as retention time and UV-Vis spectrum in the MN is mandatory to optimize the resolution of the detection of metabolites diversity, but it does not enhance the strength of annotations. In this work, using a combination of MS-guided and UV-guided fractionation was the only way to fully characterize the targeted compounds, which successfully yielded to the isolation of, among others, three isomers of LL-P880 $\beta$, including one new natural product.

The 12 isolated pyran-2-ones were found to belong to two sub-series according to their degree of insaturation of the lactone ring. These kinds of compounds are commonly found among fungi, but only the $6 S$ stereoisomers have been described as natural products, with the exception of 5,6-dihydro-(6R)-hydroxymethyl-4-methoxy-2H-pyran-2-one, the smallest C6-substituted analogue, which was previously isolated from Pestalotiopsis sydowiana [62]. In our study, all the compounds belonged to the $6 S$ series, including the new 1 , which completes the chemical family, showing that the biosynthetic route induced in these environment-related conditions was unique and stereospecific.

If monocyclic pyran-2-ones attracted much attention due to their wide distribution and chemodiversity in microbes, their biological evaluations always reported that they exert no or minor cytotoxicity nor antibacterial or antiparasitic activity, as we observed here [86,87]. Only some members were found to inhibit seed germination or plant growth, to inhibit 20S proteasome [62], to enhance the growth-stimulating action of gibberellic acid [59], or to be selectively active against some yeasts or filamentous fungi [88,89]. Their role in the chemical interaction between a fungus and its host remains then unknown, but hypotheses can be proposed. Due to their structural similarity with homoserine lactones, some synthetic and natural pyran-2-ones have been shown to act as quorum sensing inhibitors. Usually, it is considered that the longer the alkyl chain, the higher the QS inhibition activity [90]. However, it has also been discovered that pyran-2-ones, including short-branched ones, can act as signalling molecules in some bacteria such as Pseudomonas luminescens [91]. It can then be envisaged that small pyran-2-ones can be engaged in signal recognition processes and in chemical interactions between fungi and hosted bacteria inside the bivalve.

Pyran-2-ones were reported to possess potent inhibitory activities against protein tyrosine phosphatases 1B (PTP1B), as shown for two 3,4,6-trisubstituted pyran-2-one derivatives, chrysopyrones A and B, isolated from a marine Penicillium chrysogenum [80]. Protein tyrosine phosphatases (PTPs) are of importance in the regulation of a myriad of cellular signal transduction systems. They are involved in meiosis, metabolism, cellsubstrate and cell-cell adhesion, and sporulation in yeasts. They also play a role in responses to environmental changes and stresses [92]. PTP1B is the major negative regulator of insulin signalling in mammals by catalysing the phosphorylation of the insulin receptor or insulin receptor substrate [93]. Furthermore, small pyran-2-ones seem to be promising candidates for developing new PTP1B inhibitors [94-96]. The results we obtained from the in silico docking of $6 S, 1^{\prime} S$-pestalotin (10) showed that it can bind to important residues in the catalytic site of PTP1B, including some amino acids within the PTP-loop that are highly conserved in all PTPs. A whole-genome sequencing project of the cosmopolitan Mediterranean mussel (Mytilus galoprovincalis) has been conducted recently, showing that some genes with tyrosine phosphatase-related functions were present in mussels [97]. Therefore, it can be envisaged that an inhibition of PTPs is one of the mechanisms involved in the interaction of the fungus with its host. Further biological studies should be conducted to determine whether pyran-2-ones play these or other roles within molluscs. 


\section{Materials and Methods}

\subsection{Fungal Material}

Penicillium restrictum MMS417 was isolated from a sample of blue mussel Mytilus edulis collected in January 1997 at Port Giraud on the Loire estuary in France. The strain is stored at the laboratory Mer-Molécule-Santé (MMS) fungal culture collection, University of Nantes, France. The strain has been identified according to macroscopic and microscopic observations, and sequencing of the internal transcribed spacer (ITS) and $\beta$-tubulin regions of the rDNA and nucleotide BLAST search (GenBank accession number for MMS417: KU720404, KU720398).

\subsection{Culture Media Preparation}

Fungal cultures were performed in Petri dishes $(10 \mathrm{~cm}$ diameter) containing $15 \mathrm{~mL}$ of solid agar-based medium in 14 different media. Two osmotic conditions were prepared based on the presence or absence of artificial seawater ( $36 \mathrm{~g} / \mathrm{L}$ of salinity). The media compositions were as follows: DCA (dextrose $40 \mathrm{~g} / \mathrm{L}$, enzymatic digest of casein $10 \mathrm{~g} / \mathrm{L}$, agar $15 \mathrm{~g} / \mathrm{L}$, Difco); MEA (malt extract $20 \mathrm{~g} / \mathrm{L}$, peptone $1 \mathrm{~g} / \mathrm{L}$, glucose $20 \mathrm{~g} / \mathrm{L}$, agar $20 \mathrm{~g} / \mathrm{L}$ ); PDA (potato extract $4 \mathrm{~g} / \mathrm{L}$, dextrose $20 \mathrm{~g} / \mathrm{L}, \mathrm{ZnSO}_{4} \cdot 7 \mathrm{H}_{2} \mathrm{O} 0.01 \mathrm{~g} / \mathrm{L}, \mathrm{CuSO}_{4} \cdot 5 \mathrm{H}_{2} \mathrm{O} 0.005 \mathrm{~g} / \mathrm{L}$, agar $15 \mathrm{~g} / \mathrm{L}$ ); YES (yeast extract $20 \mathrm{~g} / \mathrm{L}$, sucrose $150 \mathrm{~g} / \mathrm{L}$, agar $20 \mathrm{~g} / \mathrm{L}$ ); MES (mussel extract $20 \mathrm{~g} / \mathrm{L}$, sucrose $150 \mathrm{~g} / \mathrm{L}$, agar $20 \mathrm{~g} / \mathrm{L}$ ); CYA (Czapek concentrate $10 \mathrm{~mL}$, yeast extract $5 \mathrm{~g} / \mathrm{L}$, $\mathrm{K}_{2} \mathrm{HPO}_{4} 1 \mathrm{~g} / \mathrm{L}$, sucrose $30 \mathrm{~g} / \mathrm{L}$, agar $\left.15 \mathrm{~g} / \mathrm{L}\right)$; Czapek concentrate $\left(\mathrm{NaNO}_{3} 3 \mathrm{~g} / \mathrm{L}, \mathrm{KCl}\right.$ $0.5 \mathrm{~g} / \mathrm{L}, \mathrm{MgSO}_{4} \cdot 7 \mathrm{H}_{2} \mathrm{O} 0.5 \mathrm{~g} / \mathrm{L}, \mathrm{FeSO}_{4} \cdot 7 \mathrm{H}_{2} \mathrm{O} 0.01 \mathrm{~g} / \mathrm{L}, \mathrm{ZnSO}_{4} \cdot 7 \mathrm{H}_{2} \mathrm{O} 0.01 \mathrm{~g} / \mathrm{L}, \mathrm{CuSO}_{4} \cdot 5 \mathrm{H}_{2} \mathrm{O}$ $0.05 \mathrm{~g} / \mathrm{L}$ ); $\mathrm{KMS}\left(\mathrm{MgSO}_{4} \cdot 7 \mathrm{H}_{2} \mathrm{O} 2.4 \mathrm{~g} / \mathrm{L}, \mathrm{NH}_{4} \mathrm{NO}_{3} 2.4 \mathrm{~g} / \mathrm{L}\right.$, Tris (tampon) $1.21 \mathrm{~g} / \mathrm{L}$, Agar $20 \mathrm{~g} / \mathrm{L}$ ). Details for the preparation of mussel extract are presented in a previous study [16].

\subsection{Fermentation and Extraction for OSMAC Approach}

Fungal cultures were carried out in triplicate for each medium from culture stocks of the strain stored at $-20^{\circ} \mathrm{C}$ and transplanted on Petri dishes of DCA medium 10 days before inoculation. Culture inoculations were performed by taking fragments of mycelium and conidia using a sterile Pasteur pipette and deposited at three points on the agar. Cultures were incubated at $27^{\circ} \mathrm{C}$ for 10 days under natural light. Plugs (6 mm diameter) were taken in three distinct places of cultures (at the point of central impact, on the outskirts of the colony, in the periphery but contacting an adjacent colony). The three plugs were gathered together and extracted twice with $1.5 \mathrm{~mL}$ of $\mathrm{CH}_{2} \mathrm{Cl}_{2} / \mathrm{EtOAc} 1: 1(v / v)$. Fungal mycelium and agar were extracted together to obtain both intra- and extracellular metabolites. Mixtures were sonicated for $30 \mathrm{~min}$ and filtered on $0.45 \mu \mathrm{m}$ regenerated cellulose filters (Sartorius). Organic phases were combined and evaporated to dryness, leading to an organic extract. Non-inoculated agar media were also extracted following the same protocol and used as controls (blank samples).

\subsection{HPLC-MS Analyses}

HPLC-(+)-HRESI-MS and HPLC-(+)-HRESI-MS/MS analyses were performed on a UFLC-MS (IT-TOF) Shimadzu instrument (combining ion trap and time of flight analyzers), using a Kinetex C18 column $(2.6 \mu \mathrm{m}, 2.1 \times 100 \mathrm{~mm}$, Phenomenex $)$ and following previously described conditions [41,98]. MS/MS fragmentations were obtained by applying the following parameters: energy $50 \%$, collision gas 50\%, $q$ (frequency) $0.251(45.0 \mathrm{~Hz}$ ). Precursor ion selection was performed in the range $m / z$ 150-1000. Daughter ions were measured in the range $m / z$ 100-1000 with $30 \mathrm{~ms}$ accumulation, an execution trigger set at $1 \times 10^{6}$, and an excluding dynamic range of $3 \mathrm{~s}$. Samples $(5 \mu \mathrm{L})$ were injected at the concentration of $1 \mathrm{mg} / \mathrm{mL}$ in $\mathrm{MeOH}$. MeOH blanks were injected randomly during the analysis sequences. A mixture of all extracts at the same concentration was also prepared as a quality control (QC) and regularly injected throughout the sequences. QC samples were analysed intermittently for the duration of the analytical study to assess the variance observed in the data throughout the sample preparation, data acquisition, and data pre-processing steps. 


\subsection{Data Processing}

The HPLC-(+)-HRESI-MS chromatogram raw data files were converted to *.netCDF files by using LCMS solution (version 3.60-Shimadzu). The *.netCDF files were subjected to MZmine 2 [99] for automatic peak picking. The mass detection was performed using a centroid algorithm with a noise level of $1.4^{\mathrm{E}} 4$. The chromatogram building was established using the ADAP algorithm with minimum of group size in the number of scans of 4 , a group intensity threshold of $1^{\mathrm{E}} 5$, a minimum of the highest intensity of $2.1^{\mathrm{E}} 4$ and $m / z$ tolerance of $100 \mathrm{ppm}$. The peak deconvolution was performed using the ADAP algorithm with median $\mathrm{m} / \mathrm{z}$ center calculation, a signal-to-noise threshold of 5 , minimum feature height of 1, coefficient/area threshold of 100, peak duration range from 0.08 to $23.69 \mathrm{~min}$ and retention time wavelet range from 0.01 to $0.2 \mathrm{~min}$. The chromatograms were deisotoped with an $\mathrm{m} / z$ tolerance of $0.003 \mathrm{~m} / \mathrm{z}$, retention time tolerance of $1 \mathrm{~min}$, maximum charge of 2, and the lowest $m / z$ was chosen as representative. The duplicate peaks were filtered with an $\mathrm{m} / z$ tolerance of $10 \mathrm{ppm}$ and retention time tolerance of $0.05 \mathrm{~min}$. The peak list was aligned using the Ransac aligner algorithm with an $\mathrm{m} / \mathrm{z}$ tolerance of $100 \mathrm{ppm}$, retention time tolerance of $1 \mathrm{~min}$, retention time tolerance after correction of $1 \mathrm{~min}$, Ransac interactions of 20,000, and a minimum number of points of $50.0 \%$; the value of threshold was 1 , and a linear model was chosen. The peak list rows were filtered with minimum peaks in a row of 3. Then, the gap-filling step was performed with a peak finder (multithreaded) algorithm, intensity tolerance of $30 \%, \mathrm{~m} / z$ tolerance of $5 \mathrm{ppm}$, and a retention time tolerance of $0.5 \mathrm{~min}$. All duplicate peaks were filtered by setting the $\mathrm{m} / z$ tolerance of $5 \mathrm{ppm}$ and retention time tolerance of $0.05 \mathrm{~min}$. The results were exported as a *.csv file containing all peaks observed and referenced by their mass-to-charge ratio $(\mathrm{m} / \mathrm{z})$ and retention times $\left(t_{R}\right)$ together with their respective peak areas in each sample. The generated matrix was cleaned by removing all peaks abundantly found in blank samples (culture media extracts) and technical blank samples (MeOH injections). The final data matrix corresponded to a total of 882 features $\left(m / z-t_{R}\right)$ and their respective areas in the 56 samples investigated (14 groups of four biological replicates).

\subsection{Statistical Analysis}

The data set containing 882 features was first transformed to reflect full compound production by multiplying each peak area by the extract amount of the corresponding samples. Then, the data was normalized using Pareto scaling and submitted to principal component analysis (PCA) and orthogonal projection to latent structures discriminates analysis (OPLS-DA) using SIMCA13 (UMETRICS). Variable Influence in the Projection (VIP) scores were provided by SIMCA13 software. They account for the significance of variables in the maximised groups' separation in the OPLS-DA model [100]. VIPs $>1$ were considered as significantly contributing to the separation.

\subsection{Molecular Networking}

The HPLC-(+)-HRESI-MS/MS raw data files were converted to *.netCDF files using LCMS solution (version 3.60-Shimadzu). The LC-MS/MS data was subjected to automatic peak picking using MZmine 2 [99] with the above-mentioned parameters. The peak list was exported as a *.mgf file for GNPS. The *.mgf file was subjected to the online workflow of the Global Natural Products Social molecular networking platform (https:/ / gnps.ucsd.edu, accessed on 29 June 2021) [101]. The molecular network was generated using the following settings: precursor ion mass tolerance of $2 \mathrm{Da}$ and fragment ion mass tolerance of $0.3 \mathrm{Da}$, minimum pairs cosine of 0.1 , network topK of 10 , minimum matched fragment ions of 2 , minimum cluster size of 1 ; the run MS cluster and filter precursor window tolls were turned off. It can be visualised via the following link: https://gnps.ucsd.edu/ProteoSAFe/status. jsp?task=58f66fb7b73745ce95ea632e948dc1a9, accessed on 29 June 2021. The molecular network was finally visualized using Cytoscape 3.7.1 [102]. 


\subsection{Annotation of MS Features}

Compound annotation based on MS/MS data was first performed using the GNPS platform $[84,101]$. Then, unannotated features were further identified using an alternative strategy [103]. The feature molecular formula was determined using SIRIUS3 [104] and used to mine for possible annotation in various natural product databases, such as the Dictionary of Natural Products (DNP, CRC Press), Antibase (Wiley), and KNApSAcK [105]. Fungal-related annotations were further confirmed by an exhaustive literature search for taxonomic consistency.

\subsection{Isolation and Identification of Specialized Metabolites}

Large-scale cultivation on the MES-SSW medium was performed in 54 Erlenmeyer flasks $(250 \mathrm{~mL})$, containing $50 \mathrm{~mL}$ of media. Extraction with $\mathrm{CH}_{2} \mathrm{Cl}_{2} / \mathrm{EtOAc} \mathrm{1:1} \mathrm{(v/v)}$ resulted in $11.5 \mathrm{~g}$ of extract. The extract was fractionated using silica gel vacuum liquid chromatography (VLC) eluting with mixtures of solvents with increasing polarity: hexane/EtOAc (from 100:0 to 30:70 $(v / v)$ ) to yield six fractions, then $\mathrm{CH}_{2} \mathrm{Cl}_{2} / \mathrm{MeOH}$ (from 100:0 to 0:100 $(v / v))$ to yield five fractions. Fraction F8 $(250.2 \mathrm{mg})$ was subjected to silica gel flash chromatography (column Reveleris, Silica $40 \mu \mathrm{m}, 4 \mathrm{~g}$ ) eluting with mixtures of $\mathrm{CH}_{2} \mathrm{Cl}_{2}$ /EtOAc (from 100:0 to 60:40 (v/v)), and then $\mathrm{CH}_{2} \mathrm{Cl}_{2} / \mathrm{MeOH}(50: 50(v / v)$ ) at a flow rate of $15 \mathrm{~mL} / \mathrm{min}$ to afford 13 subfractions. Fraction F8-6 (84 mg) was subjected to silica HPLC (column Luna ${ }^{\circledR} 5 \mu \mathrm{m}$, Silica, $100 \AA$, $250 \times 10 \mathrm{~mm}$ ), with a mobile phase consisting in $\mathrm{MeOH} / \mathrm{H}_{2} \mathrm{O}$ (from 3:97 to 25:75 $(v / v)$ ) at a flow rate of $4 \mathrm{~mL} / \mathrm{min}$, to yield 12 subfractions. F8-6-4 (15 mg) was fractionated by RP-pentafluorophenyl HPLC (column Kinetex ${ }^{\circledR} 5 \mu \mathrm{m}$ F5, $100 \AA, 250 \times 4.6 \mathrm{~mm})$, with the mobile phase $\mathrm{MeOH} / \mathrm{H}_{2} \mathrm{O}(20: 80(v / v))$ at a flow rate of $1 \mathrm{~mL} / \mathrm{min}$, to obtain 1 (0.5 mg), 2 (3.2 mg) and 3 (5 mg). Fraction F8-6-7 (5.5 mg) was purified by RP-pentafluorophenyl HPLC (column Kinetex ${ }^{\circledR} 5 \mu \mathrm{m}$ F5, $100 \AA$, $250 \times 4.6 \mathrm{~mm}$ ), with the mobile phase $\mathrm{MeOH} / \mathrm{H}_{2} \mathrm{O}(25: 75(v / v))$ at a flow rate of $0.5 \mathrm{~mL} / \mathrm{min}$, to afford 5 $(1.4 \mathrm{mg})$ and $6(0.7 \mathrm{mg}) . \mathrm{F} 8-7(8.7 \mathrm{mg})$ was subjected to silica HPLC (column Luna ${ }^{\circledR} 5 \mu \mathrm{m}$, Silica, $100 \AA, 250 \times 10 \mathrm{~mm}$ ), with the mobile phase $\mathrm{MeOH} / \mathrm{H}_{2} \mathrm{O}$ (from 3:97 to 25:75 (v/v)) at a flow rate of $4 \mathrm{~mL} / \mathrm{min}$, giving $4(0.7 \mathrm{mg})$. Fraction F6 $(253.8 \mathrm{mg})$ was subjected to another silica gel flash chromatography (column Macherey-Nagel, Chromabond ${ }^{\circledR}$ flash RS25-SiOH) eluting with mixtures of $\mathrm{CH}_{2} \mathrm{Cl}_{2} / \mathrm{MeOH}$ (from 100:0 to 0:100 (v/v)), at a flow rate of $20 \mathrm{~mL} / \mathrm{min}$ to afford 12 subfractions. F6-3 $(25.2 \mathrm{mg})$ was subjected to another silica gel flash chromatography eluting with mixtures of Hexane/EtOAc (from 100:0 to 0:100 $(v / v)$ ) followed by 100\% MeOH to yield six fractions. F6-3-3 (2 mg) was purified by isocratic silica HPLC (column Interchim ${ }^{\circledR} 5 \mu \mathrm{m}$, Silica, $250 \times 4.6 \mathrm{~mm}$ ), with a mobile phase of $\mathrm{CH}_{2} \mathrm{Cl}_{2} /$ EtOAc $98: 2(v / v)$ at a flow rate of $1 \mathrm{~mL} / \mathrm{min}$, to afford $7(0.9 \mathrm{mg})$.

5,6-dihydro-6S-hydroxymethyl-4-methoxy-2H-pyran-2-one (1) White amorphous powder; $[\alpha]_{D}^{20}-30$ (с 0.2, MeOH); UV $(\mathrm{MeOH}) \lambda_{\max } 238 \mathrm{~nm} ; \mathrm{CD}\left(5.06 \times 10^{-3} \mathrm{M}, \mathrm{MeOH}\right)$ $\Delta \varepsilon_{240.9}=-1.92 ;{ }^{1} \mathrm{H}$ and ${ }^{13} \mathrm{C}$ NMR data, Table $3 ;(+)$-HRESIMS $m / z 159.0652[\mathrm{M}+\mathrm{H}]^{+}$ (calculated for $\mathrm{C}_{7} \mathrm{H}_{10} \mathrm{O}_{4}, 158.058 \mathrm{Da}$ ).

6S-(1'S,2'S-dihydropentyl)-4-methoxy-5,6-dihydro-2H-pyran-2-one (2): White amorphous powder; $[\alpha]_{D}^{20} \cdot-61.71$ (c 0.58, MeOH); UV (MeOH) $\lambda_{\max } 238 \mathrm{~nm} ; \mathrm{CD}\left(1.74 \times 10^{-3} \mathrm{M}\right.$, $\mathrm{MeOH}) \Delta \varepsilon_{221.4}=+3.18, \Delta \varepsilon_{247}=-5.2 ;{ }^{1} \mathrm{H}$ and ${ }^{13} \mathrm{C}$ NMR data, Table $3 ;(+)-\mathrm{HRESIMS} m / z$ $231.1229[\mathrm{M}+\mathrm{H}]^{+}$(calculated for $\left.\mathrm{C}_{11} \mathrm{H}_{18} \mathrm{O}_{5}, 230.1156 \mathrm{Da}\right)$.

6S-(1'R,2'S-dihydropentyl)-4-methoxy-5,6-dihydro-2H-pyran-2-one (CAS 58846-08-5) (3): White amorphous powder; $[\alpha]_{D}^{20}-38.29$ (c 0.067, MeOH); UV (MeOH) $\lambda_{\max } 238 \mathrm{~nm} ; \mathrm{CD}$ $\left(4.78 \times 10^{-3} \mathrm{M}, \mathrm{MeOH}\right) \Delta \varepsilon_{222.8}+0.66, \Delta \varepsilon_{245.6}=-1.2 ;{ }^{1} \mathrm{H}$ and ${ }^{13} \mathrm{C}$ NMR data, Table 3; (+)-HRESIMS $m / z 231.1225[\mathrm{M}+\mathrm{H}]^{+}$(calculated for $\mathrm{C}_{11} \mathrm{H}_{18} \mathrm{O}_{5}, 230.1152 \mathrm{Da}$ ).

5,6-dihydro-4-methoxy-6S-(1'S,2'S-dihydroxypent-3(E)-enyl)-2H-pyran-2-one (4). White amorphous powder; UV $(\mathrm{MeOH}) \lambda_{\max } 239 \mathrm{~nm} ; \mathrm{CD}\left(8.77 \times 10^{-4} \mathrm{M}, \mathrm{MeOH}\right) \Delta \varepsilon_{223.6}=+3.01$, $\Delta \varepsilon_{246.6}=-3.75 ;{ }^{1} \mathrm{H}$ and ${ }^{13} \mathrm{C}$ NMR data, Table $3 ;(+)$-HRESIMS $m / z 229.1068[\mathrm{M}+\mathrm{H}]^{+}$ (calculated for $\mathrm{C}_{11} \mathrm{H}_{16} \mathrm{O}_{5}, 228.1 \mathrm{Da}$ ).

$\left(1^{\prime} R, 2^{\prime} S\right)-L L-P 880 \gamma$ (CAS 176780-71-5) (5): White amorphous powder; $[\alpha]_{D}^{20}+63$ (c 1.0, MeOH). UV (MeOH) $\lambda_{\max } 280 \mathrm{~nm} ; \mathrm{CD}\left(1.2 \times 10^{-3} \mathrm{M}, \mathrm{MeOH}\right) \Delta \varepsilon_{281.9}=+2.042 ;{ }^{1} \mathrm{H}$ 
and ${ }^{13} \mathrm{C}$ NMR data, Table 3; (+)-HRESIMS $m / z 229.1069[\mathrm{M}+\mathrm{H}]^{+}$(calculated for $\mathrm{C}_{11} \mathrm{H}_{16} \mathrm{O}_{5}$, 228.1 Da).

4-methoxy-6-(1'R, 2'R-dihydroxypent-3'(E)-enyl)-2H-pyran-2-one (6). White amorphous powder; $[\alpha]+55.2($ c $0.42, \mathrm{MeOH}) ; \mathrm{CD}\left(6.2 \times 10^{-3} \mathrm{M}, \mathrm{MeOH}\right) \Delta \varepsilon_{275.7}=+1.31 ; \mathrm{UV}(\mathrm{MeOH})$ $\lambda_{\max } 280 \mathrm{~nm} ;{ }^{1} \mathrm{H}$ and ${ }^{13} \mathrm{C}$ NMR data, Table 3; HRESIMS $m / z$ 227.0921 [M+H] ${ }^{+}$(calculated for $\left.\mathrm{C}_{11} \mathrm{H}_{14} \mathrm{O}_{5}, 226.0848 \mathrm{Da}\right)$.

4-methoxy-2H-pyran-2-one (7) White amorphous powder; $\mathrm{UV}(\mathrm{MeOH}) \lambda_{\max } 198,274 \mathrm{~nm}$; ${ }^{1} \mathrm{H}$ and ${ }^{13} \mathrm{C}$ NMR data, Table 3; (+)-HRESIMS $\left.m / z 127.0411[\mathrm{M}+\mathrm{H}]\right]^{+}$(calculated for $\mathrm{C}_{6} \mathrm{H}_{6} \mathrm{O}_{3}$, 126.034 Da).

\subsection{Computational Details}

All density functional theory (DFT) calculations have been performed using Gaussian 16W. A conformational analysis on both like and unlike diastereoisomers gave rise to 16 and 37 conformers, respectively. After geometry optimization at the 6-31G(d) level, NMR prediction was realized at the mpw1pw91/6-311+g(2d,p) level on each conformer. Comparison between experiment and theoretical NMR chemical shifts was achieved by calculation of the DP4 probability [71].

\subsection{Cytotoxicity Assays}

Cytotoxicity assays were carried out using the MTT assay and following the procedure previously described [41]. KB cell line was purchased from the European Collection of Animal Cell Cultures (ECACC).

\subsection{Antimicrobial Assay}

Compounds were tested for antimicrobial activity via a paper disk diffusion method, according to the Clinical and Laboratory Standards Institute (CLSI) protocol [106]. Seed cultures of Escherichia coli ATCC 25922, Pseudomonas aeruginosa ATCC 27853, Staphylococcus aureus ATCC 25923 and Enterococcus faecalis ATCC 19433 were prepared by incubating the organism for $18 \mathrm{~h}$ at $37^{\circ} \mathrm{C}$. Aliquots of overnight cultures at $1 \times 10^{7} \mathrm{CFU} / \mathrm{mL}$ were spread onto the surfaces of nutrient agar. Sterile filter disks ( $6 \mathrm{~mm}$ diameter), which were plotted with $10 \mu \mathrm{L}$ of test solution $(100 \mu \mathrm{g} / \mathrm{mL}$ in $\mathrm{EtOH} 1 \%)$, positive control (gentamicin sulfate) or vehicle only (EtOH 1\%), were added to the plates. Plates were left upright for $30 \mathrm{~min}$ at room temperature before being placed in an incubator at $37^{\circ} \mathrm{C}$ for $18 \mathrm{~h}$, and then the growth inhibition zone diameter was measured.

\subsection{Antileishmanial Activity on Leishmania infantum Axenic Amastigotes}

Leishmania infantum promastigotes (MHOM/MA/67/ITMAP-263, CNR Leishmania, Montpellier, France, expressing luciferase activity) were cultivated in RPMI 1640 medium supplemented with 10\% foetal calf serum (FCS), $2 \mathrm{mM}$ L-glutamine and antibiotics (100 U/mL penicillin and $100 \mu \mathrm{g} / \mathrm{mL}$ streptomycin) and harvested in logarithmic phase of growth by centrifugation at $900 \mathrm{~g}$ for $10 \mathrm{~min}$. The supernatant was removed carefully and was replaced by the same volume of RPMI 1640 complete medium at pH 5.4 and incubated for $24 \mathrm{~h}$ at $24^{\circ} \mathrm{C}$. The acidified promastigotes were then incubated for $24 \mathrm{~h}$ at $37^{\circ} \mathrm{C}$ in a ventilated flask to transform promastigotes into axenic amastigotes. The effects of the tested compounds on the growth of L. infantum axenic amastigotes were assessed as follows: L. infantum amastigotes were incubated at a density of $2 \times 10^{6}$ parasites $/ \mathrm{mL}$ in sterile 96-well plates with various concentrations of compounds dissolved in $\mathrm{MeOH}$ (final concentration less than $0.5 \% v / v$ ), in duplicate. Appropriate controls, DMSO, $\mathrm{MeOH}$ and amphotericin were added to each set of experiments. After a 48-h incubation period at $37^{\circ} \mathrm{C}$, each plate-well was then microscopically examined to detect any precipitate formation. To estimate the luciferase activity of axenic amastigotes, $80 \mu \mathrm{L}$ of each well were transferred to white 96-well plates, Steady Glow ${ }^{\circledR}$ reagent (Promega) was added according to the manufacturer's instructions, and plates were incubated for $2 \mathrm{~min}$. The luminescence was measured in Microbeta Luminescence Counter (PerkinElmer). Efficient concentration 
$50 \%$ ( $\mathrm{EC}_{50}$, mean of three independent experiences) was defined as the concentration of drug required to inhibit the metabolic activity of L. infantum amastigotes compared to control by $50 \%$.

\subsection{Docking}

Molecular modelling studies were performed using SYBYL-X 2.1.1 software (SYBYL-X, Tripos Associates, Inc. 1699 South Hanley Road, St. Louis, MO 63144, USA) running on an HP Z-Book Studio G3 workstation. The three-dimensional structure of compound 10 was built from a standard fragments library and optimized using the Tripos force field [107] including the electrostatic term calculated from Gasteiger and Hückel atomic charges. Powell's method available in Maximin2 procedure was used for energy minimization until the gradient value was smaller than $0.001 \mathrm{kcal} /(\mathrm{mol} \cdot \AA)$. The crystal structure of protein tyrosine phosphatase $1 \mathrm{~B}$ at a $2.4 \AA$ A resolution (PDB ID 1NWL) [108] was used as a template for docking. All water molecules were removed from the coordinates set. Flexible docking of compound 10 into the binding site was performed with GOLD software [76] using GoldScore fitness function. Finally, the selected complex was energy-minimized using Powell's method available in Maximin2 procedure with the Tripos force field and a dielectric constant of 4.0, until the gradient value reached $0.1 \mathrm{kcal} / \mathrm{mol} \cdot \AA$ A. Biovia Discovery Studio Visualizer (Dassault Systèmes BIOVIA, Discovery Studio Visualizer, v19.1.0.18287, San Diego: Dassault Systèmes, 2018) was used for graphical display.

\subsection{Electronic Circular Dichroism Measurements}

The electronic circular dichroism (ECD) spectra of small molecules were measured using a J-810 spectropolarimeter (Jasco, Japan) and a mini-quartz cell with a path length of $0.2 \mathrm{~cm}$. The spectra were recorded in the range from 200 to $300 \mathrm{~nm}$ and averaged over three scans to increase the signal-to-noise ratio (response time, $0.125 \mathrm{~s}$; data pitch $0.2 \mathrm{~nm}$, scanning speed $50 \mathrm{~nm} / \mathrm{min}$ ). The final concentrations of compounds 1-6 and reference compound 9 were $0.2 \%$ in methanol. All the spectra were corrected by subtracting the spectrum of methanol.

Supplementary Materials: The following are available online at https: / www.mdpi.com/article / 10.3390/md19070378/s1: Figure S1: Morphology of strain P. restrictum on 14 different media after 10 days of growth (seven media, two salinity); Figure S2: (a) PCA of OSMAC data in positive mode of 14 media (seven media, two salinity, four replicates) with QC (quality control); Figure S3: (a) OPLSDA score-plot of YES-SSW versus the six other SSW extracts (positive ionization, $\mathrm{n}=4$; R2X cum 0.366 and R2Y cum 0.98, Q2 cum 0.703; CV-ANOVA ( $p$-value $=5.37 \mathrm{e}-4$ ) and $(\mathrm{b})$ the corresponding S-plot: features highlighted in yellow correspond to discriminatory ions with VIP > 3; Table S1: Identification of representative characteristics ions of OPLS-DA separation YES-SSW media from other; Table S2: Dereplication from HRMS/MS data sets of the crude ethyl acetate extract of $P$. restrictum MMS417 on MES-SSW medium; Figure S4: Annotation of cluster 3-light blue nodes represent features with values VIP > 1; Figure S5: LC-(+)-ESIHRMS spectrum and UV-Vis spectrum of compound 1 with corresponding structure; Figure S6: LC-(+)-ESI HRMS spectrum and UV-Vis spectrum of compound 2 with corresponding structure; Figure S7: LC-(+)-ESIHRMS spectrum and UV-Vis spectrum of compound 3 with corresponding structure; Figure S8: LC-(+)-ESIHRMS spectrum and UV-Vis spectrum of compound 4 with corresponding structure; Figure S9: LC- $( \pm)$-ESIHRMS spectrum and UV-Vis spectrum of compound 5 with corresponding structure; Figure S10: LC-(+)ESIHRMS spectrum and UV-Vis spectrum of compound 6 with corresponding structure; Figure S11: LC-(+)-ESIHRMS spectrum and UV-Vis spectrum of compound 7 with corresponding structure; Figure S12-S50: 1D and 2D NMR spectra of compounds 1-7.

Author Contributions: Conceptualization, S.B., O.G.; methodology and investigation, V.-T.L., T.R.d.P., F.F., S.B.-D., N.C., E.G., C.L., G.G.-J.; data analyses, S.B., N.C., S.B.-D., C.L., G.G.-J., O.G., writing, reviewing and editing V.-T.L., S.B., N.C., C.L., G.G.-J., O.G. All authors have read and agreed to the published version of the manuscript.

Funding: This research received no external funding. 
Data Availability Statement: Further data are available on request from the corresponding authors.

Acknowledgments: The authors acknowledge the Vietnam International Education Development program for a Ph.D. grant for V.T. Le. The authors are grateful to Pascal Richomme from laboratory SONAS, University of Angers and Muriel Duflos and Pascal Marchand from laboratory IiciMed, University of Nantes for NMR analyses, and Marylène Chollet-Krugler from laboratory ISCR UMR CNRS 6226, University of Rennes 1, for optical rotations determinations. The authors also aknowledge the IMPACT Core Facility of Biogenouest, UMR CNRS 6286 Fonctionnalité et Ingénierie des Protéines (UFIP), Universiy of Nantes, for ECD experiments, and the Corsaire-ThalassOMICS Metabolomics Core Facility of Biogenouest, for LC-HRMS/MS analyses and automated dereplication.

Conflicts of Interest: The authors declare no conflict of interest.

\section{References}

1. Gosling, E. Phylogeny and evolution of bivalve molluscs. In Marine Bivalve Molluscs; John Wiley \& Sons, Ltd.: Hoboken, NJ, USA, 2015; pp. 1-11.

2. Pereira, C.S.; Possas, C.D.A.; Viana, C.M.; Rodrigues, D.D.P. Características de Vibrio parahaemolyticus isolados de mexilhões (Perna perna) comercializados em Niterói, Rio de Janeiro. Rev. Soc. Bras. Med. Trop. 2007, 40, 56-59. [CrossRef]

3. Leal, M.C.M.R.; Cammarota, M.C.; Freire, D.M.G.; Sant'Anna, G.L., Jr. Hydrolytic enzymes as coadjuvants in the anaerobic treatment of dairy wastewaters. Braz. J. Chem. Eng. 2002, 19, 175-180. [CrossRef]

4. Potasman, I.; Paz, A.; Odeh, M. Infectious outbreaks associated with bivalve shellfish consumption: A worldwide perspective. Clin. Infect. Dis. 2002, 35, 921-928. [CrossRef] [PubMed]

5. Lévesque, B.; Barthe, C.; Dixon, B.R.D.R.; Parrington, L.J.P.J.; Martin, D.; Doidge, B.; Proulx, J.-F.-F.; Murphy, D. Microbiological quality of blue mussels (Mytilus edulis) in Nunavik, Quebec: A pilot study. Can. J. Microbiol. 2010, 56, 968-977. [CrossRef] [PubMed]

6. Sallenave-Namont, C.; Pouchus, Y.F.; Robiou du Pont, T.; Lassus, P.; Verbist, J.F. Toxigenic saprophytic fungi in marine shellfish farming areas. Mycopathologia 2000, 149, 21-25. [CrossRef] [PubMed]

7. Zvereva, L.V.; Vysotskaya, M.A. Filamentous fungi associated with bivalve mollusks from polluted biotopes of Ussuriiskii bay, sea of Japan. Russ. J. Mar. Biol. 2005, 31, 382-385. [CrossRef]

8. Santos, A.; Hauser-Davis, R.A.; Santos, M.J.S.; De Simone, S.G. Potentially toxic filamentous fungi associated to the economically important Nodipecten nodosus (Linnaeus, 1758) scallop farmed in southeastern Rio de Janeiro, Brazil. Mar. Pollut. Bull. 2017, 115, 75-79. [CrossRef] [PubMed]

9. Jones, E.B.G.; Pang, K.-L.; Abdel-Wahab, M.A.; Scholz, B.; Hyde, K.D.; Boekhout, T.; Ebel, R.; Rateb, M.E.; Henderson, L.; Sakayaroj, J.; et al. An online resource for marine fungi. Fungal Div. 2019, 96, 347-433. [CrossRef]

10. Spiteller, P. Chemical ecology of fungi. Nat. Prod. Rep. 2015, 32, 971-993. [CrossRef]

11. Rédou, V.; Vallet, M.; Meslet-Cladière, L.; Kumar, A.; Pang, K.-L.; Pouchus, Y.F.; Barbier, G.; Grovel, O.; Bertrand, S.; Prado, S.; et al. Chapter 4. Marine fungi. In The Marine Microbiome-An Untold Resource of Biodiversity and Biotechnological Potential, 1st ed.; Stal, L.J., Cretoiu, M.S., Eds.; Springer International Publishing: Cham, Switzerland, 2016; pp. 99-154.

12. Antipova, T.V.; Zhelifonova, V.P.; Baskunov, B.P.; Kochkina, G.A.; Ozerskaya, S.M.; Kozlovskii, A.G. Exometabolites the Penicillium Fungi isolated from various high-latitude ecosystems. Microbiology 2018, 87, 642-651. [CrossRef]

13. Vansteelandt, M.; Kerzaon, I.; Blanchet, E.; Fossi Tankoua, O.; Robiou Du Pont, T.; Joubert, Y.; Monteau, F.; Le Bizec, B.; Frisvad, J.C.; Pouchus, Y.F.; et al. Patulin and secondary metabolite production by marine-derived Penicillium strains. Fungal Biol. 2012, 116, 954-961. [CrossRef]

14. Petit, K.E.; Mondeguer, F.; Roquebert, M.F.; Biard, J.F.; Pouchus, Y.F. Detection of griseofulvin in a marine strain of Penicillium waksmanii by ion trap mass spectrometry. J. Microbiol. Methods 2004, 58, 59-65. [CrossRef] [PubMed]

15. Matallah-Boutiba, A.; Ruiz, N.; Sallenave-Namont, C.; Grovel, O.; Amiard, J.-C.; Pouchus, Y.F.; Boutiba, Z. Screening for toxigenic marine-derived fungi in Algerian mussels and their immediate environment. Aquaculture 2012, 342-343, 75-79. [CrossRef]

16. Geiger, M.; Guitton, Y.; Vansteelandt, M.; Kerzaon, I.; Blanchet, E.; Robiou du Pont, T.; Frisvad, J.C.; Hess, P.; Pouchus, Y.F.; Grovel, O. Cytotoxicity and mycotoxin production of shellfish-derived Penicillium spp., a risk for shellfish consumers. Lett. Appl. Microbiol. 2013, 57, 385-392. [CrossRef]

17. Bertrand, S.; Bohni, N.; Schnee, S.; Schumpp, O.; Gindro, K.; Wolfender, J.-L. Metabolite induction via microorganism co-culture: A potential way to enhance chemical diversity for drug discovery. Biotechnol. Adv. 2014, 32, 1180-1204. [CrossRef]

18. Romano, S.; Jackson, S.; Patry, S.; Dobson, A. Extending the “One Strain Many Compounds” (OSMAC) principle to marine microorganisms. Mar. Drugs 2018, 16, 244. [CrossRef] [PubMed]

19. Overy, D.P.; Zidorn, C.; Petersen, B.O.; Duus, J.Ø.; Dalsgaard, P.W.; Larsen, T.O.; Phipps, R.K. Medium dependant production of corymbiferone a novel product from Penicillium hordei cultured on plant tissue agar. Tetrahedron Lett. 2005, 46, 3225-3228. [CrossRef]

20. Overy, D.P.; Blunt, J.W. Corymbiferan lactones from Penicillium hordei: Stimulation of novel phenolic metabolites using plant tissue media. J. Nat. Prod. 2004, 67, 1850-1853. [CrossRef] 
21. Osman, M.; Stigloher, C.; Mueller, M.J.; Waller, F. An improved growth medium for enhanced inoculum production of the plant growth-promoting fungus Serendipita indica. Plant Methods 2020, 16, 39. [CrossRef] [PubMed]

22. Cagigal, E.F.-M.; Sánchez, A.C. Influence of the culture media and the organic matter in the growth of Paxillus ammoniavirescens (Contu \& Dessi). Mycobiology 2017, 45, 172-177. [CrossRef]

23. Overy, D.P.; Smedsgaard, J.; Frisvad, J.C.; Phipps, R.K.; Thrane, U. Host-derived media used as a predictor for low abundant, in planta metabolite production from necrotrophic fungi. J. Appl. Microbiol. 2006, 101, 1292-1300. [CrossRef]

24. Medina, M.L.; Kiernan, U.A.; Francisco, W.A. Proteomic analysis of rutin-induced secreted proteins from Aspergillus flavus. Fungal Genet. Biol. 2004, 41, 327-335. [CrossRef]

25. Stajic, M.; Persky, L.; Cohen, E.; Hadar, Y.; Brceski, I.; Wasser, S.P.; Nevo, E. Screening of laccase, manganese peroxidase, and versatile peroxidase activities of the genus Pleurotus in media with some raw plant materials as carbon sources. Appl. Biochem. Biotechnol. 2004, 117, 155-164. [CrossRef]

26. Dhillon, B.; Feau, N.; Aerts, A.L.; Beauseigle, S.; Bernier, L.; Copeland, A.; Foster, A.; Gill, N.; Henrissat, B.; Herath, P.; et al. Horizontal gene transfer and gene dosage drives adaptation to wood colonization in a tree pathogen. Proc. Natl. Acad. Sci. USA 2015, 112, 3451-3456. [CrossRef]

27. Torrado, A.; Vázquez, J.-A.; Prieto, M.-Á.; Fuciños, P.; Montemayor, M.-I.; Pastrana, L.; González, M.-P.; Murado, M.-Á. Amylase production by Aspergillus oryzae in a solid-state bioreactor with fed-batch operation using mussel processing wastewaters as feeding medium. J. Chem Technol. Biotechnol. 2013, 88, 226-236. [CrossRef]

28. Sankaran, S.; Khanal, S.K.; Jasti, N.; Jin, B.; Pometto, A.L.; Van Leeuwen, J.H. Use of filamentous fungi for wastewater treatment and production of high Value fungal byproducts: A review. Crit. Rev. Environ. Sci. Technol. 2010, 40, 400-449. [CrossRef]

29. Amado, I.R.; Vázquez, J.A. Mussel processing wastewater: A low-cost substrate for the production of astaxanthin by Xanthophyllomyces dendrorhous. Microb. Cell Factories 2015, 14, 177. [CrossRef] [PubMed]

30. Pastrana, L.M.; Gonzalez, M.P.; Murado, M.A. Production of gibberellic acid from mussel processing wastes in submerged batch culture. Bioresour. Technol. 1993, 45, 213-221. [CrossRef]

31. González, M.P.; Siso, M.I.G.; Murado, M.A.; Pastrana, L.; Montemayor, M.I.; Mirón, J. Depuration and valuation of musselprocessing wastes. Characterization of amylolytic postincubates from different species grown on an effluent. Bioresour. Technol. 1992, 42, 133-140. [CrossRef]

32. Murado, M.A.; González, M.P.; Pastrana, L. Mussel processing wastes as a fermentation substrate. In Fisheries Processing; Springer: Boston, MA, USA, 1994; pp. 311-343.

33. Nicoletti, R.; Stefano, M.D. Penicillium restrictum as an antagonist of plant pathogenic fungi. Dyn. Biochem. Process Biotechnol. Mol. Biol. 2012, 6, 61-69.

34. Sankhala, R. Metabolic products of Penicillium restrictum. Indian J. Exp. Biol. 1968, 6, 57-58.

35. Schwartz, R.E.; Dufresne, C.; Flor, J.E.; Kempf, A.J.; Wilson, K.E.; Lam, T.; Onishi, J.; Milligan, J.; Fromtling, R.A.; Abruzzo, G.K. Restricticin, a novel glycine-containing antifungal agent. J. Antibiot. 1991, 44, 463-471. [CrossRef]

36. Hensens, O.D.; Wichmann, C.F.; Liesch, J.M.; Vanmiddlesworth, F.L.; Wilson, K.E.; Schwartz, R.E. Structure elucidation of restricticin, a novel antifungal agent from Penicillium restrictum. Tetrahedron 1991, 47, 3915-3924. [CrossRef]

37. Raistrick, H.; Rice, F.A.H. 2,3-Dihydro-3,6-dihydroxy-2-methyl-4-pyrone and curvularin from Penicillium gilmanii. J. Chem. Soc. C Org. 1971, 3069. [CrossRef] [PubMed]

38. Jackson, M.; Karwowski, J.P.; Humphrey, P.E.; Kohl, W.L.; Barlow, G.J.; Tanaka, S.K. Calbistrins, novel antifungal agents produced by Penicillium restrictum. I. Production, taxonomy of the producing organism and biological activity. J. Antibiot. 1993, 46, 34-38. [CrossRef]

39. Brill, G.M.; Chen, R.H.; Rasmussen, R.R.; Whittern, D.N.; McAlpine, J.B. Calbistrins, novel antifungal agents produced by Penicillium restrictum. II. Isolation and elucidation of structure. J. Antibiot. 1993, 46, 39-47. [CrossRef] [PubMed]

40. Martín, A.; Jurado, M.; Rodríguez, M.; Núñez, F.; Córdoba, J.J. Characterization of molds from dry-cured meat products and their metabolites by micellar electrokinetic capillary electrophoresis and random amplified polymorphic DNA PCR. J. Food Prot. 2004, 67, 2234-2239. [CrossRef] [PubMed]

41. Hoang, T.P.T.; Roullier, C.; Boumard, M.-C.; Robiou du Pont, T.; Nazih, H.; Gallard, J.-F.; Pouchus, Y.F.; Beniddir, M.A.; Grovel, O. Metabolomics-driven discovery of meroterpenoids from a mussel-derived Penicillium ubiquetum. J. Nat. Prod. 2018, 81, $2501-2511$. [CrossRef] [PubMed]

42. Scott, A.I. Chapter 4-O- and S-heteroaromatic compounds. In Interpretation of the Ultraviolet Spectra of natural Products; Scott, A.I., Ed.; International Series of Monographs on Organic Chemistry; Pergamon: Oxford, UK, 1964; pp. 135-164.

43. Collett, L.A.; Davies-Coleman, M.T.; Rivett, D.E.A. Naturally occurring 6-substituted 5,6-dihydro- $\alpha$-pyrones. In Fortschritte der Chemie Organischer Naturstoffe/Progress in the Chemistry of Organic Natural Products; Springer: Vienna, Austria, 1998; pp. $181-209$.

44. McGahren, W.J.; Ellestad, G.A.; Morton, G.O.; Kunstmann, M.P.; Mullen, P. New fungal lactone, LL-P880 $\beta$, and a new pyrone, LL-880 $\gamma$, from a Penicillium species. J. Org. Chem. 1973, 38, 3542-3544. [CrossRef]

45. Gorst-Allman, C.; Steyn, P. Biosynthesis of 5, 6-dihydro-4-methoxy-2H-pyran-2-one in Penicillium italicum. S. Afr. J. Chem. 1983, $36,83-84$.

46. Kimura, Y.; Hamasaki, T.; Nakajima, H. Stereochemistry and biological activities of LL-P880 $\gamma$, a pestalotin analogue, produced by Penicillium citreo-viride. Agric. Biol. Chem. 1986, 50, 1649-1650. [CrossRef] 
47. Sakai, K.; Chiba, H.; Kaneto, R.; Sakamoto, M.; Okamura, K.; Tone, H. Mer-NF8054A and X, novel antifungal steroids, isolated from Aspergillus sp. J. Antibiot. 1994, 47, 591-594. [CrossRef] [PubMed]

48. Li, J.; Wang, Y.; Hao, X.; Li, S.; Jia, J.; Guan, Y.; Peng, Z.; Bi, H.; Xiao, C.; Cen, S.; et al. Broad-spectrum antiviral natural products from the marine-Derived Penicillium sp. IMB17-046. Molecules 2019, 24, 2821. [CrossRef] [PubMed]

49. Nakano, H.; Hara, M.; Yamashita, Y.; Ando, K.; Shuto, K. Paxisterol, a new analgesic sterol without anti-inflammation activity from Penicillium. J. Antibiot. 1988, 41, 409-410. [CrossRef]

50. Okabe, M.; Sugita, T.; Kinoshita, K.; Koyama, K. Macrolides from a marine-derived fungus, Penicillium meleagrinum var. viridiflavum, showing synergistic effects with fluconazole against azole-resistant Candida albicans. J. Nat. Prod. 2016, 79, 1208-1212. [CrossRef]

51. Gao, N.; Shang, Z.-C.; Yu, P.; Luo, J.; Jian, K.-L.; Kong, L.-Y.; Yang, M.-H. Alkaloids from the endophytic fungus Penicillium brefeldianum and their cytotoxic activities. Chin. Chem. Lett. 2017, 28, 1194-1199. [CrossRef]

52. Ishimaru, T.; Harada, S.; Tsuboya, S. TAN-1446 Exhibiting Anti-Angiotensin II Activity and its Manufacture with Penicillium restrictum. Japan Patent JP 05041994, 23 February 1993.

53. Larsen, T.O.; Frisvad, J.C.; Christophersen, C. Arabenoic acid (verrucolone), a major chemical indicator of Penicillium verrucosum. Biochem. Syst. Ecol. 1998, 26, 463-465. [CrossRef]

54. Isaac, B.G.; Ayer, S.W.; Stonard, R.J. Arabenoic acid, a natural product herbicide of fungal origin. J. Antibiot. 1991, 44, 793-794. [CrossRef]

55. Matsumura, M.; Miki, S.; Oyama, K.; Tsuchida, M.; Yamamoto, K. New Agricultural/Horticultural Bactericide. Japan Patent JP2007280064A, 29 October 2009.

56. Nose, H.; Seki, A.; Yaguchi, T.; Hosoya, A.; Sasaki, T.; Hoshiko, S.; Shomura, T. PF1163A and B, new antifungal antibiotics produced by Penicillium sp. I. Taxonomy of producing strain, fermentation, isolation and biological activities. J. Antibiot. 2000, 53, 33-37. [CrossRef]

57. Sasaki, T.; Nose, H.; Hosoya, A.; Yoshida, S.; Kawaguchi, M.; Watanabe, T.; Usui, T.; Ohtsuka, Y.; Shomura, T.; Takano, S.; et al. PF1163A and B, new antifungal antibiotics Produced by Penicilium sp. II. Physico-chemical properties and structure elucidation. J. Antibiot. 2000, 53, 38-44. [CrossRef] [PubMed]

58. Ellestad, G.A.; McGahren, W.J.; Kunstmann, M.P. Structure of a new fungal lactone, LL-P880 $\alpha$, from an unidentified Penicillium species. J. Org. Chem. 1972, 37, 2045-2047. [CrossRef] [PubMed]

59. Kimura, Y.; Tamura, S. Isolation and structure of pestalotin, a gibberellin synergist from Pestalotia cryptomeriaecola. Agric. Biol. Chem. 1972, 36, 1925-1930. [CrossRef]

60. Kimura, Y.; McGahren, W.J.; Suzuki, A.; Tamura, S. Structure of a new fungal pyrone, from an unidentified Penicillium sp. Agric. Biol. Chem. 1978, 42, 1625-1626. [CrossRef]

61. Evidente, A.; Zonno, M.C.; Andolfi, A.; Troise, C.; Cimmino, A.; Vurro, M. Phytotoxic $\alpha$-pyrones produced by Pestalotiopsis guepinii, the causal agent of hazelnut twig blight. J. Antibiot. 2012, 65, 203-206. [CrossRef] [PubMed]

62. Xia, X.; Kim, S.; Liu, C.; Shim, S. Secondary metabolites produced by an endophytic fungus Pestalotiopsis sydowiana and their 20S proteasome inhibitory activities. Molecules 2016, 21, 944. [CrossRef]

63. Pospisil, J.; Marko, I.E. Metathesis-based synthesis of 3-methoxy $\alpha, \beta$-unsaturated lactones: Total synthesis of (R)-kavain and of the C1-C6 fragment of jerangolid D. Tetrahedron Lett. 2008, 49, 1523-1526. [CrossRef]

64. Zhao, Q.; Wang, C.-X.; Yu, Y.; Wang, G.-Q.; Zheng, Q.-C.; Chen, G.-D.; Lian, Y.-Y.; Lin, F.; Guo, L.-D.; Gao, H. Nodulisporipyrones A-D, new bioactive $\alpha$-pyrone derivatives from Nodulisporium sp. J. Asian Nat. Prod. Res. 2015, 17, 567-575. [CrossRef] [PubMed]

65. Kirihata, M.; Ohta, K.; Ichimoto, I.; Ueda, H. Total synthesis of (6S,1S,2R)-6-(1,2-Dihydroxypentyl)-4-methoxy-5,6-dihydropyrane2-one (LL-P880ß) and its C6-epimer, a fungal metabolite from Penicillium sp. Agric. Biol. Chem. 1990, 54, 2401-2405. [CrossRef]

66. Rönsberg, D.; Debbab, A.; Mándi, A.; Wray, V.; Dai, H.; Kurtán, T.; Proksch, P.; Aly, A.H. Secondary metabolites from the endophytic fungus Pestalotiopsis virgatula isolated from the mangrove plant Sonneratia caseolaris. Tetrahedron Lett. 2013, 54, 3256-3259. [CrossRef]

67. Kirihata, M.; Ohe, M.; Ichimoto, I.; Ueda, H. Stereoselective synthesis of unnatural stereoisomers of LL-P880 $\beta$ and LL-P880 $\gamma$, pestalotin analogues from Penicillium sp. Biosci. Biotechnol. Biochem. 1992, 56, 1825-1828. [CrossRef]

68. Kirihata, M.; Kamihisa, Y.; Ichimoto, I.; Ueda, H. Stereoselective synthesis of of $\left(6 \mathrm{R}, 1^{\prime} \mathrm{R}, 2^{\prime} \mathrm{S}\right)-$ and $\left(6 \mathrm{~S}, 1^{\prime} \mathrm{R}, 2^{\prime} \mathrm{S}\right)-\mathrm{LL}-\mathrm{P} 880 \beta$, stereoisomers of the fungal metabolite from Penicillium strains. Chem. Express 1992, 7, 837.

69. Kirihata, M.; Ohe, M.; Ichimoto, I.; Kimura, Y. Synthesis and biological activity of LL-P880 $\gamma$ and its analogues. Biosci. Biotechnol. Biochem. 1996, 60, 677-679. [CrossRef]

70. Wang, J.; Xu, C.-C.; Tang, H.; Su, L.; Chou, Y.; Soong, K.; Li, J.; Zhuang, C.-L.; Luo, Y.-P.; Zhang, W. Osteoclastogenesis inhibitory polyketides from the sponge-associated fungus Xylaria feejeensis. Chem. Biodivers. 2018, 15, e1800358. [CrossRef]

71. Smith, S.G.; Goodman, J.M. Assigning stereochemistry to single diastereoisomers by GIAO NMR Calculation: The DP4 probability. J. Am. Chem. Soc. 2010, 132, 12946-12959. [CrossRef] [PubMed]

72. Ermanis, K.; Parkes, K.E.B.; Agback, T.; Goodman, J.M. The optimal DFT approach in DP4 NMR structure analysis-pushing the limits of relative configuration elucidation. Org. Biomol. Chem. 2019, 17, 5886-5890. [CrossRef]

73. Rao, K.V.; Reddy, G.C.S. A new reaction of patulin. J. Nat. Prod. 1989, 52, 1376-1378. [CrossRef] 
74. Ji, S.; Li, Z.; Song, W.; Wang, Y.; Liang, W.; Li, K.; Tang, S.; Wang, Q.; Qiao, X.; Zhou, D.; et al. Bioactive constituents of Glycyrrhiza uralensis (Licorice): Discovery of the effective components of a traditional herbal medicine. J. Nat. Prod. 2016, 79, 281-292. [CrossRef]

75. Han, W.; Cai, J.; Zhong, W.; Xu, G.; Wang, F.; Tian, X.; Zhou, X.; Liu, Q.; Liu, Y.; Wang, J. Protein tyrosine phosphatase 1B (PTP1B) inhibitors from the deep-sea fungus Penicillium chrysogenum SCSIO 07007. Bioorganic Chem. 2020, 96, 103646. [CrossRef] [PubMed]

76. Jones, G.; Willett, P.; Glen, R.C.; Leach, A.R.; Taylor, R. Development and validation of a genetic algorithm for flexible docking 1 1Edited by F. E. Cohen. J. Mol. Biol. 1997, 267, 727-748. [CrossRef]

77. Tonks, N.K. PTP1B: From the sidelines to the front lines! FEBS Lett. 2003, 546, 140-148. [CrossRef]

78. Sharma, B.; Xie, L.; Yang, F.; Wang, W.; Zhou, Q.; Xiang, M.; Zhou, S.; Lv, W.; Jia, Y.; Pokhrel, L.; et al. Recent advance on PTP1B inhibitors and their biomedical applications. Eur. J. Med. Chem. 2020, 199, 112376. [CrossRef] [PubMed]

79. Brandão, T.A.S.; Hengge, A.C.; Johnson, S.J. Insights into the reaction of protein-tyrosine phosphatase 1B. J. Biol. Chem. 2010, 285, 15874-15883. [CrossRef] [PubMed]

80. Igboeli, H.A.; Marchbank, D.H.; Correa, H.; Overy, D.; Kerr, R.G. Discovery of primarolides A and B from marine fungus Asteromyces cruciatus using osmotic stress and treatment with suberoylanilide hydroxamic acid. Mar. Drugs 2019, $17,435$. [CrossRef] [PubMed]

81. Auckloo, B.N.; Pan, C.; Akhter, N.; Wu, B.; Wu, X.; He, S. Stress-driven discovery of novel cryptic antibiotics from a marine fungus Penicillium sp. BB1122. Front. Microbiol. 2017, 8, 1450. [CrossRef]

82. Overy, D.; Correa, H.; Roullier, C.; Chi, W.-C.; Pang, K.-L.; Rateb, M.; Ebel, R.; Shang, Z.; Capon, R.; Bills, G.; et al. Does osmotic stress affect natural product expression in fungi? Mar. Drugs 2017, 15, 254. [CrossRef] [PubMed]

83. Wang, Y.; Lu, Z.; Sun, K.; Zhu, W. Effects of high salt stress on secondary metabolite production in the marine-derived fungus Spicaria elegans. Mar. Drugs 2011, 9, 535-542. [CrossRef]

84. Wang, M.; Carver, J.J.; Phelan, V.V.; Sanchez, L.M.; Garg, N.; Peng, Y.; Nguyen, D.D.; Watrous, J.; Kapono, C.A.; Luzzatto-Knaan, T.; et al. Sharing and community curation of mass spectrometry data with Global Natural Products Social molecular networking. Nat. Biotechnol. 2016, 34, 828-837. [CrossRef]

85. Yang, J.Y.; Sanchez, L.M.; Rath, C.M.; Liu, X.; Boudreau, P.D.; Bruns, N.; Glukhov, E.; Wodtke, A.; de Felicio, R.; Fenner, A.; et al. Molecular networking as a dereplication strategy. J. Nat. Prod. 2013, 76, 1686-1699. [CrossRef]

86. Trisuwan, K.; Rukachaisirikul, V.; Borwornwiriyapan, K.; Phongpaichit, S.; Sakayaroj, J. Pyrone derivatives from the soil fungus Fusarium solani PSU-RSPG37. Phytochem. Lett. 2013, 6, 495-497. [CrossRef]

87. Liu, D.; Li, X.-M.; Meng, L.; Li, C.-S.; Gao, S.-S.; Shang, Z.; Proksch, P.; Huang, C.-G.; Wang, B.-G. Nigerapyrones A-H, $\alpha-$ pyrone derivatives from the marine mangrove-derived endophytic fungus Aspergillus niger MA-132. J. Nat. Prod. 2011, 74, $1787-1791$. [CrossRef] [PubMed]

88. Zhao, T.; Xu, L.-L.; Zhang, Y.; Lin, Z.-H.; Xia, T.; Yang, D.-F.; Chen, Y.-M.; Yang, X.-L. Three new $\alpha$-pyrone derivatives from the plant endophytic fungus Penicillium ochrochloronthe and their antibacterial, antifungal, and cytotoxic activities. J. Asian Nat. Prod. Res. 2019, 21, 851-858. [CrossRef] [PubMed]

89. Evidente, A.; Cabras, A.; Maddau, L.; Serra, S.; Andolfi, A.; Motta, A. Viridepyronone, a new antifungal 6-substituted 2H-pyran2-one produced byTrichoderma virid. J. Agric. Food. Chem. 2003, 51, 6957-6960. [CrossRef] [PubMed]

90. Park, S.; Kim, H.-S.; Ok, K.; Kim, Y.; Park, H.-D.; Byun, Y. Design, synthesis and biological evaluation of 4-(alkyloxy)-6-methyl2H-pyran-2-one derivatives as quorum sensing inhibitors. Bioorganic Med. Chem. Lett. 2015, 25, 2913-2917. [CrossRef]

91. Brachmann, A.O.; Brameyer, S.; Kresovic, D.; Hitkova, I.; Kopp, Y.; Manske, C.; Schubert, K.; Bode, H.B.; Heermann, R. Pyrones as bacterial signaling molecules. Nat. Chem. Biol. 2013, 9, 573-578. [CrossRef] [PubMed]

92. Ghosh, A.; Servin, J.A.; Park, G.; Borkovich, K.A. Global analysis of serine/threonine and tyrosine protein phosphatase catalytic subunit genes in Neurospora crassa reveals interplay between phosphatases and the p38 mitogen-activated protein kinase. G3 Genes Genomes Genet. 2014, 4, 349-365. [CrossRef]

93. Phaedra, E.; Athina, G.; Anthi, P. PTP1b Inhibition, A Promising Approach for the Treatment of Diabetes Type II. Curr. Top. Med. Chem. 2019, 19, 246-263. [CrossRef]

94. Lee, D.-S.; Jang, J.-H.; Ko, W.; Kim, K.-S.; Sohn, J.; Kang, M.-S.; Ahn, J.; Kim, Y.-C.; Oh, H. PTP1B inhibitory and anti-Inflammatory effects of secondary metabolites isolated from the marine-derived fungus Penicillium sp. JF-55. Mar. Drugs 2013, 11, 1409-1426. [CrossRef]

95. Hohmann, C.; Schneider, K.; Bruntner, C.; Brown, R.; Jones, A.L.; Goodfellow, M.; Krämer, M.; Imhoff, J.F.; Nicholson, G.; Fiedler, H.-P.; et al. Albidopyrone, a new $\alpha$-pyrone-containing metabolite from marine-derived Streptomyces sp. NTK 227. J. Antibiot. 2009, 62, 75-79. [CrossRef]

96. Helaly, S.; Schneider, K.; Nachtigall, J.; Vikineswary, S.; Tan, G.Y.A.; Zinecker, H.; Imhoff, J.F.; Süssmuth, R.D.; Fiedler, H.P. Gombapyrones, new $\alpha$-pyrone metabolites produced by Streptomyces griseoruber Acta 3662. J. Antibiot. 2009, 62, 445-452. [CrossRef] [PubMed]

97. Murgarella, M.; Puiu, D.; Novoa, B.; Figueras, A.; Posada, D.; Canchaya, C. A first insight into the genome of the filter-feeder mussel Mytilus galloprovincialis. PLoS ONE 2016, 11, e0151561. [CrossRef]

98. Roullier, C.; Bertrand, S.; Blanchet, E.; Peigné, M.; Robiou du Pont, T.; Guitton, Y.; Pouchus, Y.F.; Grovel, O. Time dependency of biosynthetic pathways and chemodiversity: An LC-MS metabolomic study of marine-sourced Penicillium. Mar. Drugs 2016, 14, 103. [CrossRef] [PubMed] 
99. Pluskal, T.; Castillo, S.; Villar-Briones, A.; Oresic, M. MZmine 2: Modular framework for processing, visualizing, and analyzing mass spectrometry-based molecular profile data. BMC Bioinform. 2010, 11, 395. [CrossRef] [PubMed]

100. Bylesjö, M.; Rantalainen, M.; Cloarec, O.; Nicholson, J.K.; Holmes, E.; Trygg, J. OPLS discriminant analysis: Combining the strengths of PLS-DA and SIMCA classification. J. Chemom. 2006, 20, 341-351. [CrossRef]

101. Aron, A.T.; Gentry, E.; McPhail, K.L.; Nothias, L.F.; Nothias-Esposito, M.l.; Bouslimani, A.; Petras, D.; Gauglitz, J.M.; Sikora, N.; Vargas, F.; et al. Reproducible molecular networking of untargeted mass spectrometry data using GNPS. Nat. Protoc. 2020, 15, 1954-1991. [CrossRef]

102. Shannon, P.; Markiel, A.; Ozier, O.; Baliga, N.S.; Wang, J.T.; Ramage, D.; Amin, N.; Schwikowski, B.; Ideker, T. Cytoscape: A software environment for integrated models of biomolecular interaction networks. Genome Res. 2003, 13, 2498-2504. [CrossRef]

103. Bertrand, S.; Guitton, Y.; Roullier, C. Successes and pitfalls in automated dereplication strategy using liquid chromatography coupled to mass spectrometry data: A CASMI 2016 experience. Phytochem. Lett. 2017, 21, 297-305. [CrossRef]

104. Böcker, S.; Letzel, M.C.; Lipták, Z.; Pervukhin, A. SIRIUS: Decomposing isotope patterns for metabolite identification. Bioinformatics 2009, 25, 218-224. [CrossRef] [PubMed]

105. Shinbo, Y.; Nakamura, Y.; Altaf-Ul-Amin, M.; Asahi, H.; Kurokawa, K.; Arita, M.; Saito, K.; Ohta, D.; Shibata, D.; Kanaya, S. KNApSAcK: A comprehensive species-metabolite relationship database. In Plant Metabolomics; Saito, K., Dixon, R., Willmitzer, L., Eds.; Biotechnology in Agriculture and Forestry; Springer: Berlin/Heidelberg, Germany, 2006; Volume 57, pp. 165-181. Available online: http:/ / kanaya.naist.jp/KNApSAcK/ (accessed on 28 June 2021).

106. Wikler, M.A. Performance Standards for Antimicrobial Disk Susceptibility Tests; Approved Standard-Ninth edition; (M2-A9); CLSI: Wayne, PA, USA, 2006.

107. Clark, M.; Cramer, R.D.; Van Opdenbosch, N. Validation of the general purpose tripos 5.2 force field. J. Comput. Chem. 1989, 10, 982-1012. [CrossRef]

108. Erlanson, D.A.; McDowell, R.S.; He, M.M.; Randal, M.; Simmons, R.L.; Kung, J.; Waight, A.; Hansen, S.K. Discovery of a new phosphotyrosine mimetic for PTP1B using breakaway tethering. J. Am. Chem. Soc. 2003, 125, 5602-5603. [CrossRef] 